Submitted Nov 5, 2009; ACCEPTED By APJ APRIL 6, 2010

Preprint typeset using LTEX style emulateapj v. 12/01/06

\title{
THE ENVIRONMENTAL DEPENDENCE OF THE LUMINOSITY-SIZE RELATION FOR GALAXIES
}

\author{
PREeTHi B. NAIR* \\ Department of Astronomy \& Astrophysics, University of Toronto, 50 St. George Street, Toronto, ON, M5S 3H4.
}

\begin{abstract}
SIDNEY VAN DEN BERGH
Dominion Astrophysical Observatory, Herzberg Institute of Astrophysics, National Research Council of Canada, 5071 West Saanich Road, Victoria, British Columbia, Canada V9E 2E7
\end{abstract}

\author{
ROBERTO G. ABRAHAM \\ Department of Astronomy \& Astrophysics, University of Toronto, 50 St. George Street, Toronto, ON, M5S 3H4. \\ Submitted Nov 5, 2009; Accepted by ApJ April 6, 2010
}

\begin{abstract}
We have examined the luminosity-size relationship as a function of environment for 12150 SDSS galaxies with precise visual classifications from the catalog of Nair \& Abraham (2010). Our analysis is subdivided into investigations of early-type galaxies and late-type galaxies. Early-type galaxies reveal a surprisingly tight luminosity-size relation. The dispersion in luminosity about the fiducial relation is only $\sim 0.14 \mathrm{dex}(0.35 \mathrm{mag})$, even though the sample contains galaxies which differ by a factor of almost 100 in luminosity. The dispersion about the luminosity-size relation is comparable to the dispersion about the fundamental plane, even though the luminosity-size relation is fundamentally simpler and computed using purely photometric parameters. The key contributors to the dispersion about the luminosity-size relation are found to be color and central concentration. Expanding our analysis to the full range of morphological types, we show that the slope, zero point, and scatter about the luminosity-size relation is independent of environmental density. Our study thus indicates that whatever process is building galaxies is doing so in a way that preserves fundamental scaling laws even as the typical luminosity of galaxies changes with environment. However, the distribution of galaxies along the luminosity-size relation is found to be strongly dependent on galaxy environment. This variation is in the sense that, at a given morphology, larger and more luminous galaxies are rarer in sparser environments. Our analysis of late-type galaxy morphologies reveals that scatter increases towards later Hubble types. Taken together, these results place strong constraints on conventional hierarchical models in which galaxies are built up in an essentially stochastic way.
\end{abstract}

Subject headings: galaxies: fundamental parameters, galaxies: photometry, galaxies: morphology

\section{INTRODUCTION}

In hierarchical models the formation of galaxies is driven by multiple mergers and complicated feed-back effects. As a result the star-formation and mass-building history of individual galaxies can differ in a multitude of ways. However, a common feature of these models is that the end-product of this process is generally a system which lies on (or at least near) a fundamental scaling relation e.g. the fundamental plane for elliptical galaxies (Bovlan-Kolchin et al. 2006; Robertson et al. 2006b; Ciotti et al. 2007; Covington et al. 2008), or the Tully-Fisher relation for spiral galaxies (Dalcanton et al. 1997)). In some models this agreement is somewhat artificial, because the form of the scaling relation is taken as an input parameter, but in other cases it emerges because some fundamental relations are closely linked to simple underlying physics (e.g. the Faber-Jackson relation emerges from the virial theorem coupled with assumptions about massto-light ratio being a weak function of mass).

How can measurements of the fundamental scaling relations of galaxies best be used to constrain ideas for galaxy

* Present address: INAF - Astronomical Observatory of Bologna, Via Ranzani 1, I - 40127 Bologna, ITALY

Electronic address: preethi.nair@oabo.inaf.it

Electronic address: sidney.vandenbergh@nrc-cnrc.gov.ca

Electronic address: abraham@astro.utoronto.ca formation? It is arguable that the most direct way forward is simply to measure how the form of these relations changes with environment. Environment is the central parameter in hierarchical models, since in this picture the ultimate fate of a galaxy is determined mainly by its merger history, and the rate of merging is accelerated in richer environments, which are expected to host the oldest and most massive galaxies at any time (Mo \& White 1996). It is perhaps even more interesting to measure the scatter about the fundamental relations as a function of environment, because even if a relation's mathematical form is set by simple physics, the process by which a galaxy winds up on that relation is governed by random processes. A galaxy's stochastic merger history sets the timing over which it builds, but its final state is the product of a complex interplay between a large number of parameters, such as its initial mass, angular momentum and dark halo concentration, not to mention feedback to the intergalactic medium, so it would be surprising if the scatter about the fundamental relations were very small.

In a recent paper van den Bergh (2008) found that the luminosity-diameter relation provides the tightest of all the purely photometric correlations used to characterize galaxies. This fact was first noted by Giuricin et al. (1988), who also claim to have detected "appreciable differences in the galaxyluminosity relationships for different clusters". On the other 
hand, Girardi et al. (1991) found no significant differences between the luminosity-diameter relations in a variety of environments. Gavazzi et al. (1996) and van den Bergh (2008) have reached similar conclusions, albeit with small samples, and both suggest that galaxies are best viewed as complex systems linked by a single fundamental property, namely stellar mass. This viewpoint is supported by Disney et al. (2008), who concluded the same thing on the basis of a principal component analysis of data from a sample of galaxies selected from a large blind survey of neutral hydrogen gas emission.

On the other hand, recent work (Shen et al. 2003; Kauffmann et al. 2003b; Bernardi et al. 2003; Desroches et al. 2007; Hyde \& Bernardi 2008) using much larger samples drawn from the SDSS (York et al. 2000) have shown the size-luminosity and size-mass relations exhibit curvature for both early and late type galaxies. In addition, Bernardi et al. (2007); Bernardi (2009); Lauer et al. (2007); von der Linden et al. (2007); Liu et al. (2008); Coenda \& Muriel (2009) have found that the central or brightest cluster galaxies (BCGs) exhibit a steeper sizeluminosity relation than field early type galaxies, though Guo et al. (2009) and Weinmann et al. (2009) do not find the trend.

The works just described are based on either small $(<1000)$ samples of visually classified objects or much larger samples $(>50,000)$ with very broad morphological segregations based on measurements of central concentration. The recent publication of the detailed morphological catalog of Nair \& Abraham (2010) offers us an opportunity to revisit the question of the environmental dependence of the galactic scaling relations as a function of morphology and environment using samples with high-precision classifications that are an order of magnitude larger than those available to previous authors. Our aim in the present paper is to determine whether the form and scatter of the luminosity-size relation for galaxies remains constant as a function of environment. We will explore a range of Hubble types from $\mathrm{E}$ to $\mathrm{Sc}$, but our main emphasis will be on early-type systems. This is because in hierarchical models elliptical galaxies are the endproduct of major mergers, so they are a crucible for testing the central idea of merger-driven evolution. Early-type galaxies also provide us with the closest connection with existing work on this subject, because they dominate the populations of rich clusters, and most previous work on the environmental dependence of the luminosity-size relationship has focused on simple comparisons between rich clusters and the field. Our own sample differs from this earlier work in that it does not focus on rich clusters, and instead spans a range of environments from sparse regions in the field up to systems that could best be characterized as poor-intermediate clusters. In our study there are only 700 galaxies in clusters with $\log \left(M_{\text {halo }} / M_{\odot}\right)>14.0$ and our data includes galaxies from only 47 clusters with more than 50 members (described below).

The plan for this paper follows: Our sample is described in Section 2 followed by our measurements of the fiducial luminosity-size relationships in Section 3. The dependence of these relationships on environment is explored in Section 4 Section 5 looks into the importance of color and central concentration on scaling relations. Section 6 investigates the luminosity-size relationship for the brightest cluster galaxies (BCGs) in comparison to field and cluster satellite galaxies. Our results are discussed in Section 8 and conclusions are presented in Section 9. We compare the two SDSS size measures, R(90) and R(dev), in Appendix A and compare the publicly available environment estimates used in this paper in the Appendix B. Throughout this paper we assume a flat dark energy-dominated cosmology with $h=0.7$ and $\Omega_{\Lambda}=0.7$. All magnitudes quoted are in the AB system.

\section{SAMPLE}

We use the sample of 14034 visually classified bright galaxies from Nair \& Abraham (2010) (NA10) to investigate the luminosity-size relation in the local universe. The reader is referred to the NA10 catalog paper for details, but in summary the local sample is derived from the SDSS DR4 release which covers 6670 square degrees in the $u^{\prime}, g^{\prime}, r^{\prime}, i^{\prime}, z^{\prime}$ (3553 $\AA$, $4686 \AA, 6166 \AA, 7480 \AA, 8932 \AA$ ) bands. The galaxies are selected from the spectroscopic main galaxy sample described in Strauss et al. (2002). The DR4 best photometry catalogs were used to select all objects with an extinction corrected $g$ 'band magnitude brighter than 16.0 at redshifts between 0.01 and 0.1. Visual classification of the entire sample was carried out by one of the authors (PN) using the Carnegie Atlas of Galaxies (Sandage \& Bedke 1994) as a visual training set, in consultation with the Third Reference Catalog of Bright Galaxies (de Vaucouleurs et al. 1991, RC3), along with images for many fiducial objects obtained using the IPAC NED database. The classifications are found to agree with those from the RC3, with a mean deviation of 1.2 T-types for the $\sim 1700$ galaxies in common to both samples. It is important to note that although our sample probes a wide range in mass and luminosities, it has magnitude cuts imposed and is not volume-limited. However, this sample can be used to understand the influence of detailed morphology as well as to compare the different metrics used to measure morphology such as the Sérsic index, central concentration and color. For this paper, we use the SDSS derived g-band Petrosian R(90) radii and absolute magnitude (Strauss et al. 2002), corrected for galactic extinction (Schlegel et al. 1998) and K-corrected to $\mathrm{z}=0.0$ using $K$-correct (Blanton et al. 2005b). The effect of seeing correction on the Petrosian measure of sizes is negligible (see Appendix A). It should be noted that Petrosian magnitudes are not ideal because they capture a typedependent fraction of the total light of a galaxy (Graham et al. 2005; Bernardi et al. 2009). However, unlike de Vaucouleur sizes, they are not sensitive to central concentrations within a type (see Appendix A). We use stellar masses derived by Kauffmann et al. (2003a). The sample analyzed here is a clean sub-sample of 12150 objects with no projection effects by satellites, foreground objects or nearby stars. This leads to the exclusion of $\sim 400 \mathrm{BCG}$ and some nearby satellite galaxies as defined by Yang et al. (2007). Environment estimates are available for the entire sample. The environmental estimates used will be described next.

\subsection{Measures of Environment}

There are multiple metrics that have been used to describe the environment of galaxies in the Sloan Survey, such as the over-density estimates used by Blanton et al. (2005a), an Nth nearest neighbor approach used by Baldry et al. (2006), and group catalog algorithms used by Yang et al. (2007). What follows is a short summary of these three main techniques, and some justification for our decision to only use two of these (the Nth nearest neighbour aproach of Baldry et al. (2006), and the group catalog approach of Yang et al. (2007) in the present paper. The reader is referred to the original papers cited above for details. 

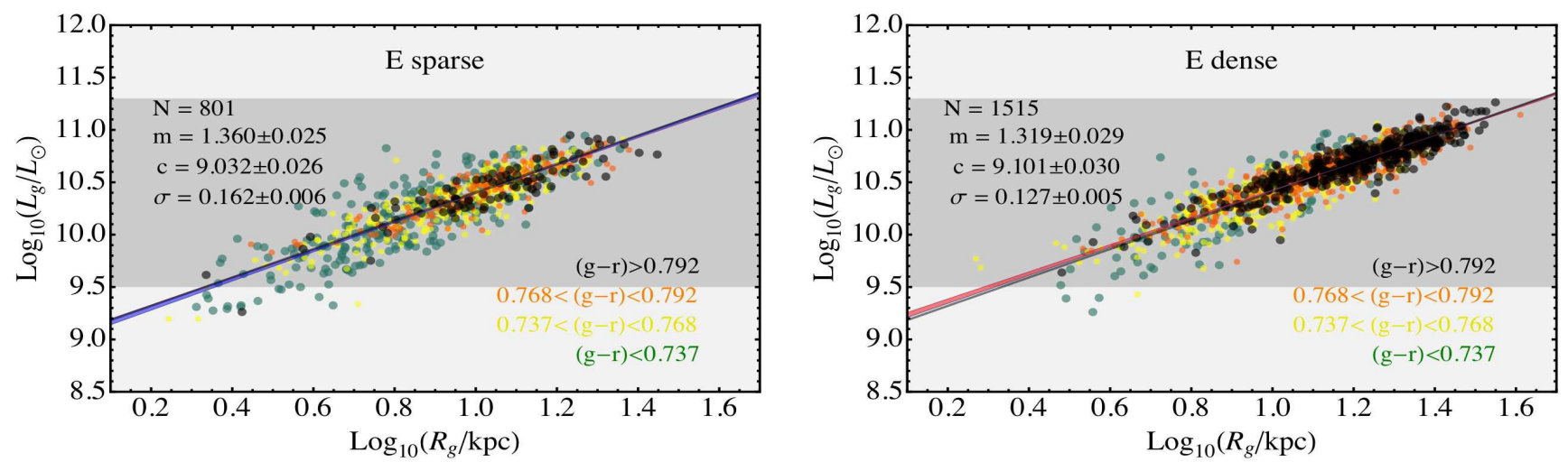

FIG. 1.- The g-band luminosity versus size plot, for galaxies in the sparsest regions (left-hand panel) and densest regions (right-hand panel) in our sample. Luminosity is in solar units, and size is defined using the Petrosian $\mathrm{R}(90)$ radius in the rest frame. Sparse regions are defined as $\mathrm{N} \leq 3$ and Baldry rho $<-0.32$, while dense regions are defined as $\mathrm{N} \geq 3$ and Baldry rho $>-0.32$. The points are keyed to $g-r$ color quartiles. Black points are the reddest galaxies in each panel, followed by orange, then yellow with green points being the bluest galaxies. Note the remarkably low scatter about the luminosity-size relationship. See text for details.

The environmental overdensity $\rho$ estimated by the New York Value Added Galaxy Catalog (NYU-VAGC) group (Blanton et al. 2005a) was calculated for each spectroscopically targeted galaxy in the SDSS $(14.5<\mathrm{r}<17.77$ and $0.05<\mathrm{z}<0.22$ ) For each galaxy, neighbors were counted in the 'SDSS imaging in the magnitude range corresponding to $\mathbf{M}^{*} \pm 1$ mag (passively evolved and $\mathrm{K}$-corrected as for an early-type galaxy) and within $5 \mathrm{~h}^{-1} \mathrm{Mpc}$ (transverse, proper)', excluding the target galaxy. The count was weighted to recover the estimated overdensity averaged over a spherical three-dimensional Gaussian window $e^{-r^{2}} / 2 a^{2}$ with a radius of $\mathrm{a}=1 \mathrm{~h}^{-1} \mathrm{Mpc}$ (proper).

Baldry et al. (2006) measured the environmental density for SDSS galaxies with an extinction corrected r-band magnitude brighter than 18 for spectroscopically selected galaxies between $0.005<\mathrm{z}<0.3$ and photometrically selected galaxies with surface brightness $18.5<\mu_{r, 50}<24.0$. The density is defined as $\Sigma=N /\left(\pi d_{N}^{2}\right)$, where $d_{N}$ is the projected comoving distance (in Mpc) to the Nth nearest neighbour (within \pm 1000 $\mathrm{km} / \mathrm{s}$ if a spectroscopic redshift was available or else with photometric redshift errors within the 95 percent confidence limit). A best estimate density (to account for spectroscopic incompleteness) was obtained by calculating the average density for $\mathrm{N}=4$ and $\mathrm{N}=5$ with spectroscopically confirmed members only and with the entire sample. The mean $\log \Sigma$ for our sample is -0.32 .

Yang et al. (2007) used an iterative halo-based group finder on the NYU-VAGC SDSS catalog for objects with an extinction corrected r-band magnitude brighter than 18.0 mag and $0.01<\mathrm{z}<0.2$ with a redshift confidence $C_{z}>0.7$. Tentative group members were identified using a modified friendsof-friends algorithm. The group members were used to determine the group center, size, mass and velocity dispersion. New group memberships were determined iteratively based on the halo properties. The final catalog yields additional information identifying the brightest galaxy in the group (BCG), the most massive galaxy in the group (both used as proxies for central galaxies), estimated group mass, group luminosity and halo mass. ${ }^{1}$

As described in the Appendix B, when comparing the different environmental estimates we find the Blanton et al.

\footnotetext{
${ }^{1}$ For groups with only one member, the brightest galaxy and most massive galaxy flags are also set.
}

(2005a) measurements are biased against small galaxies in dense environments in comparison to the other two estimate. Hence, in this paper we use the Baldry et al. (2006) and Yang et al. (2007) estimates to define galaxies in clusters and in the field. We use the group occupation number $\mathrm{N}$ as a proxy for environment. Group mass, luminosity or distance from the group center can also be used. In the Appendix we show a comparison between the Baldry et al. (2006) environmental estimator and the group occupation number determined by Yang et al. (2007). We find that for groups with more than 7 spectroscopically confirmed members, the Yang and Baldry estimates of dense environments are consistent. However, galaxies considered to be more isolated by Yang $(\mathrm{N}<3)$ can occur in a range of environments as determined by Baldry et al. (2006). This emphasizes the difficulties in determining environment (small scales vs group scales) and which measure matters (Blanton \& Berlind 2007; Deng et al. 2009). We are primarily interested in relative evolution between field and cluster galaxies and hence define low density regions as galaxies with $\mathrm{N} \leq 2$ and Baldry rho $<-0.32$ while high density regions are defined as galaxies in groups with more than two members $\mathrm{N}>2$ and Baldry rho $>-0.32$ (the mean $\mathrm{N}$ and Log $\Sigma$ for our sample). When we consider BCGs versus satellite galaxies in clusters, we do not include groups with occupation numbers less than 2 unlike Weinmann et al. (2009) who assume satellite galaxies were not detected for those 'central' galaxies with $\mathrm{N}<2$.

\section{LUMINOSITY-SIZE RELATIONS}

The luminosity-size relationship for early type galaxies is shown Figure 1 for sparse and dense environments. The points are keyed to color quartiles for each Hubble type where black points are the reddest galaxies in each panel and green points the bluest galaxies. Orange and yellow points are the intermediate quartiles. The shaded region in each plot shows the luminosity range spanned by elliptical galaxies in dense regions. This figure clearly shows that the luminosity-size relationship of elliptical galaxies defines a remarkable tight relationship. For elliptical galaxies as a whole, the data are well-represented by a power-law of the form:

$$
\log \left(L_{g}\right)=(9.03 \pm 0.02)+(1.38 \pm 0.01) \times \log \left(R_{p 90}\right)
$$



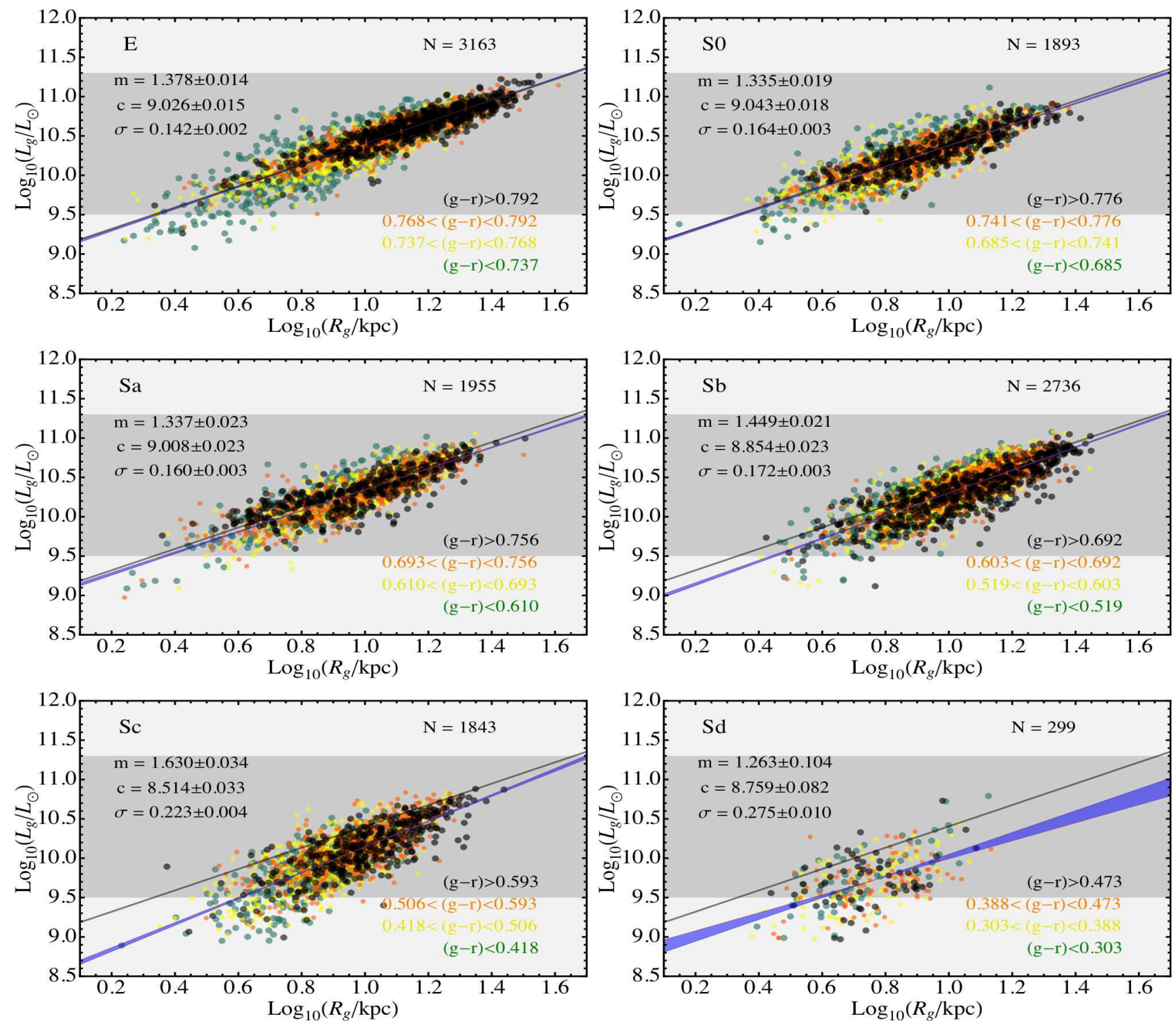

FIG. 2.- The g-band luminosity versus size for a range of galaxy types. Luminosity is in solar units, and size is defined using Petrosian R(90) radius in the rest frame. Each panel isolates a specific Hubble type Nair \& Abraham (2010), as noted in the text. The points are keyed to $g-r$ color quartiles. Black points are the reddest galaxies in each panel, followed by orange, then yellow with green points being the bluest galaxies. The black line shows the relation for elliptical galaxies. The blue lines/regions in each panel denote the best fit relation for each particular Hubble type.

This equation defines the fiducial line shown in black on both panels of Figure 1. The colored sector in each panel of this figure illustrates the $\pm 1 \sigma$ range of permissible slopes. For high-density environments (rho $>-0.32$ and $\mathrm{N}>2$ ) the data for $\mathrm{E}+\mathrm{E} / \mathrm{S} 0$ galaxies are well-represented by the equation:

$$
\log \left(L_{g}\right)=(9.10 \pm 0.03)+(1.32 \pm 0.03) \times \log \left(R_{p 90}\right)
$$

The corresponding relation for $\mathrm{E}+\mathrm{E} / \mathrm{S} 0$ galaxies in lowdensity environments ( rho $<-0.32$ and $\mathrm{N} \leq 2$ ) is given by:

$$
\log \left(L_{g}\right)=(9.03 \pm 0.03)+(1.36 \pm 0.03) \times \log \left(R_{p 90}\right)
$$

We therefore find that the luminosity-radius relations for $E$ galaxies are statistically indistinguishable for high and low density regions. However, inspection of Figure 1 and of Table 1 shows that the the RMS dispersion about these relations do show an environmental dependence ${ }^{2}$. The dispersion

\footnotetext{
${ }^{2}$ Note that dispersions in Table 1 are in dex, not magnitudes.
}

around the fiducial relation is $\sigma=0.13$ dex in dense environments, and $\sigma=0.16 \mathrm{dex}$ in sparse environments (equivalent to $0.32 \mathrm{mag}$ and $0.40 \mathrm{mag}$, respectively). In agreement with work by Gallazzi et al. (2006), who investigated the environmental dependence of the Faber-Jackson relation with SDSS galaxies (though with a larger dispersion of $\sigma=0.58$ mag), we find the reddest elliptical galaxies lie predominantly in dense regions, and that these show a smaller dispersion than do corresponding systems that are blue (and which lie in sparser regions). What is surprising, however, is that the dispersion about the fiducial relationship is so small.

For the elliptical galaxy sample as a whole (including galaxies in all environments) shown in Figure 2, the dispersion is only $0.14 \mathrm{dex}(0.35 \mathrm{mag})$. The significance of this very small dispersion about the luminosity-size relationship is best appreciated by comparing it with the corresponding scatter about the fundamental plane. Bernardi et al. (2006) have derived the dispersion in the distance from the fundamental plane in $g$-band for early-type galaxies, and their Table 5 shows a dispersion of $\sigma=0.345$ mag in high-density 
regions and $\sigma=0.355$ mag in low-density regions, defined over a sample spanning a similar dynamic range of luminosity to that being examined here.

Therefore the tightness of the luminosity-size relationship for elliptical galaxies is comparable to the scatter about the fundamental plane. This is in spite of the fact that the luminosity-size relationship is defined on the basis of purely photometric parameters, with no spectroscopic information needed (aside from a redshift). The luminosity-size relation exhibits a scatter (0.35 mag) that is almost half the typical scatter in the Faber-Jackson relation $(\sim 0.58 \mathrm{mag}$, Gallazzi et al. (2006)).

The remarkable tightness of the luminosity-size relationship should not blind us to the existence of some significant scatter about the fiducial line given by Equation (1), and it is interesting to consider the source of this dispersion. We will have more to say about this in Section 5, and for now only note that it is apparent from Figure 1 that the reddest elliptical galaxies (black points) exhibit the tightest relation between size and luminosity (in both dense and sparse environments). This suggests that star-formation history (contributing through flux-weighted stellar population age) is an important component of the scatter, as has been found by Shankar \& Bernardi (2009). We will show later that the dispersion in the relationship can be decreased even further by incorporating central concentration as a third parameter, suggesting that both star formation history and structure are important contributors to the scatter.

Expanding our consideration to later Hubble types, Figure 2 shows the distributions of galaxy size vs luminosity in panels which segregate galaxies into the following broad type bins: $\mathrm{E}+\mathrm{E} / \mathrm{S} 0, \mathrm{~S} 0+\mathrm{S} 0 / \mathrm{a}, \mathrm{Sa}+\mathrm{Sab}, \mathrm{Sb}+\mathrm{Sbc}, \mathrm{Sc}+\mathrm{Scd}, \mathrm{Sd}+\mathrm{Sm}$. Linear least-squares fits to the relations shown in this figure are included in Table 1. In the remainder of this paper we will drop the sub-type nomenclature when referring to galaxies, and thus when we refer to elliptical galaxies we refer to $\mathrm{E}+$ E/S0 galaxies. It is seen from Figure 2 and Table 1 that both the slope and the dispersion in the best-fitting relationships increases with morphological type (i.e with later morphologies), with fairly little dependence on the color of the galaxy. To allow the various panels to be compared more easily, the best fit relation for elliptical galaxies is shown in every panel of Figure 2 as a black line. The blue lines/regions in each panel denote the best fit relation for each particular Hubble type. Our aim is to allow the reader to determine from inspection of this figure whether the slope of the best-fitting line differs from that defined by the elliptical galaxy population. To this end, for each Hubble type we have plotted the lines with maximum and minimum slope consistent with the $1 \sigma$ uncertainties (determined by 100 bootstraps), and shaded the region between these lines in light blue.

It is apparent that as Hubble type increases an increasing fraction of objects fall below the relationship for elliptical galaxies defined by Eqn. (1). As has already been noted, the values of the slope and intercept of the best linear fits to the $L_{g}$ vs. $R_{p 90}$ data for each Hubble type are presented in Table 1 The uncertainties on the tabulated quantities presented in this table were all calculated using the statistical bootstrap method (Efron \& Tibshirani 1994) with 100 iterations. This table also contains fit data for sub-categories of galaxies grouped by environment, as described in the next section.

\section{EFFECT OF ENVIRONMENT}

In our discussion of Figure 1 it was already clear that environment did not change the form of the fiducial luminositysize relationship for early-type galaxies, but that it did play a significant (but not overwhelming) role in increasing (slightly) the dispersion about the fiducial relationship. It is also clear that environment plays a role in skewing the earlytype population as a whole toward brighter galaxies as the density increases. In this section we examine whether similar trends are seen in the sample as a whole. Before doing this, it is first important to note that the dependence of galactic properties on the environment of galaxies has recently been studied by various groups using different metrics to define environment and morphology. Blanton et al. (2003), Blanton et al. (2005a) and Hogg et al. (2004) find that luminous galaxies reside preferentially in high-density regions and that blue galaxies are mainly located in low-density regions. Coenda \& Muriel (2009) find that bright galaxies preferentially inhabit cluster centers, and that early-type galaxies in the field have lower luminosities than do their counterparts in clusters.

In Figure 3 we now investigate these effects in greater detail using the environmental density parameters described earlier. (Note that these density estimates are all contained in the NA10 catalog). For simplicity, we once again adopt the strategy used in Figure 1 and divide the data for each morphological type into two bins corresponding to regions of aboveaverage, or below-average, environmental density.

Figure 3 illustrates how the trends shown in the previous figure depend on environment, and the main message seems to be that environment has little effect on the shape of the fiducial relationships for each type, although, as with elliptical galaxies, there appears to be a somewhat greater scatter about the relationship in spare environments. For example, the dispersion of the data around the fiducial relationship is found to be $\sigma=0.22 \mathrm{dex}$ for Sc galaxies. This value is much larger than that for $\mathrm{E}(\sigma=0.14 \mathrm{dex})$ and $\mathrm{Sa}(\sigma=0.16 \mathrm{dex})$ galaxies. The relationship between scatter and galaxy color noted in our discussion of Figure 1 turns out to be generally true, as we see that the scatter is smallest amongst red galaxies for every Hubble type.

An arguably more important effect of environment is also shown in Figure 3. Environment seems to curtail the range of sizes exhibited by certain Hubble types, while leaving the form of the luminosity-size relation unchanged. This is made clear by inspection of the final two columns in Table 1, which lists the size range spanned by galaxies from the 10\%-90\% quantiles. The absence of large ellipticals in the field has already been noted, but another effect of environment is to limit the number of small Sc galaxies in rich environments. This effect will be discussed further in the next section.

\section{EFFECT OF COLOR AND CONCENTRATION}

Figure 4 shows galaxies defined to be on the red sequence or the blue cloud (as defined by Baldry et al. (2004)) for each morphological bin keyed to the central concentration ${ }^{3}$ of the galaxy. A number of striking trends are obvious from this figure, and from the summary of the trends given in Table 1 Firstly, it is seen that the scatter in the luminosity-size relation for elliptical galaxies is somewhat smaller on the red sequence

\footnotetext{
${ }^{3}$ Defined as the ratio of flux within an inner and outer elliptical aperture determined from the sky-subtracted, intensity-weighted, second-order moment of the image. The major and minor axes of the outer aperture are normalized so that the total area within the ellipse is the area of the galaxy. The inner aperture is defined by scaling these axes down by a factor of 3 .
} 

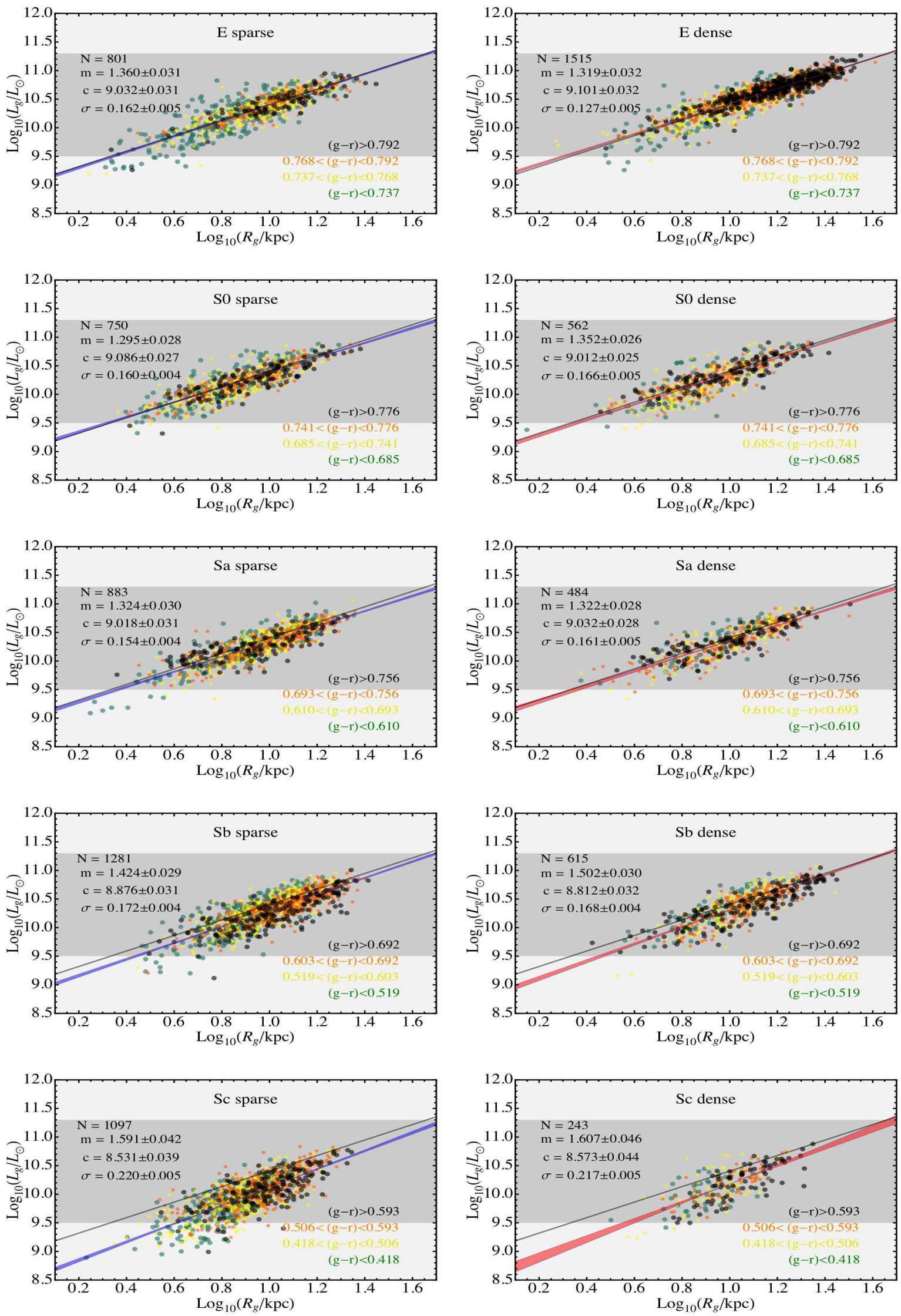

FIG. 3.- g-band luminosity versus g-band Petrosian R(90) radius in kpc per morphological type in low density regions (left) and high density regions (right). The points are keyed to $g-r$ color quartiles. Black points are the reddest galaxies in each panel, followed by orange, then yellow with green points being the bluest galaxies. The black line shows the relation for elliptical galaxies. The blue lines/regions and red lines/regions in each panel denote the best fit relation for each particular Hubble type in sparse and dense environments respectively. 

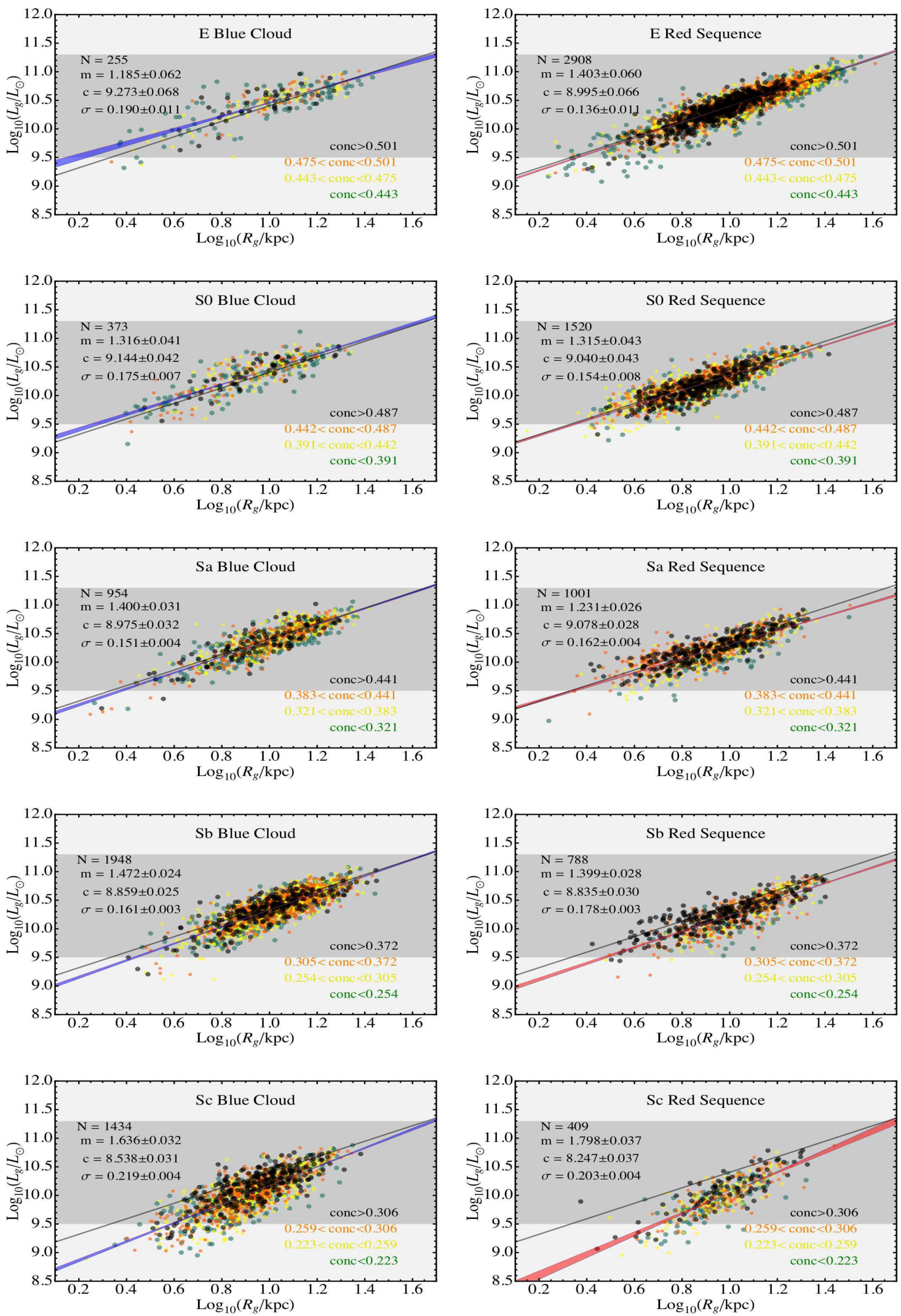

FIG. 4.- g-band luminosity versus g-band Petrosian R(90) radius in kpc as a function of morphological type in the blue cloud (left) and red sequence (right) keyed to central concentration. The most concentrated galaxies are shown in black, and the least concentrated are shown in green. Orange and Yellow are intermediate concentrations with orange points sampling the larger quartile. The black line shows the relation for elliptical galaxies. The blue lines/regions and red lines/regions in each panel denote the best fit relation for each particular Hubble type in the blue cloud and red sequence respectively. 

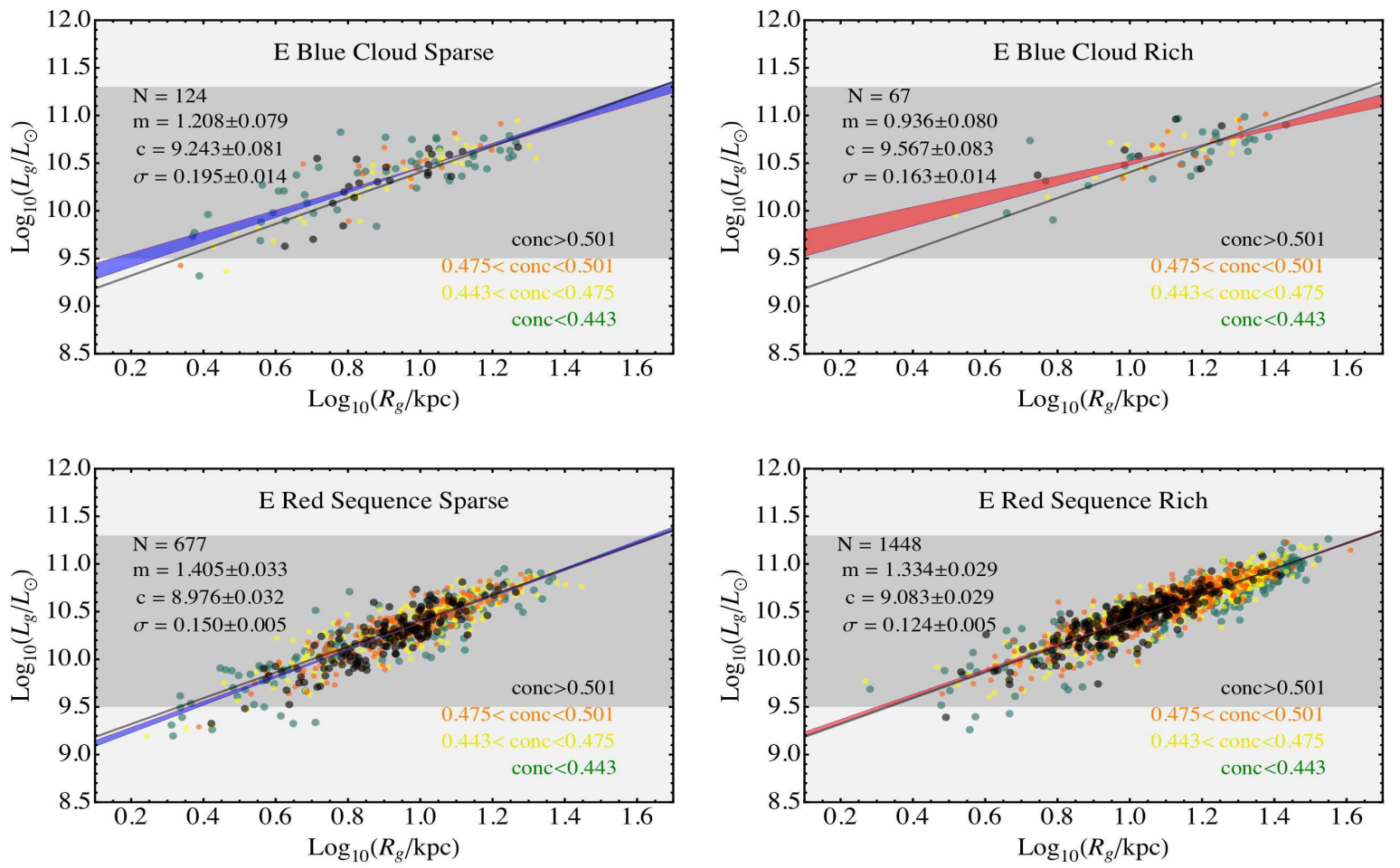

FIG. 5.- g-band luminosity versus g-band Petrosian R(90) radius in kpc per morphological type on the blue cloud (top) and red sequence (bottom) in low (left) and high density (right) regions keyed to central concentration. The most concentrated galaxies are shown in black, followed by orange and yellow with the least concentrated galaxies shown in green.

than in the blue cloud. From Figure 1 we find that color does matter in terms of increasing the dispersion, and Table 1 indicates that the scatter is $\sim 50 \%$ larger for early-type galaxies in the blue cloud than on the red sequence. Secondly, we find increasing central concentration also has the effect of decreasing the dispersion and making modest changes in the slopes and intercepts of the fiducial relation for elliptical galaxies. This trend is explored in greater detail in Table 2, which investigates the luminosity-size relationships for earlytype galaxies as a function of concentration. We see from this table that the slope and intercept of the luminosity-size relation for high-concentration early-type galaxies appears to be significantly different than for low-concentration early-types. This is manifested as a mild stratification in the plot symbol colors in the top-right panel of Figure 4. Our suspicion is that the trends with concentration may well be due to merger history, following on ideas given by Kormendy et al. (2009), who find that the merger history of elliptical galaxies determines the presence of internal structures like cores and excess light in the central regions of galaxies as well as their Sérsic in$\mathrm{dex}^{4}$. On the other hand, since the general trends remain similar and the trends with concentration seem a second-order effect, our results (and the work of (van den Bergh 2008)) seem to suggest that the overall dimensions of early type galaxies (i.e. sizes measured at a faint isophote, well beyond the inner regions) do not seem to be greatly affected by mergers. The luminosity-size relation for $\mathrm{Sa}$ - Sc galaxies does show a mild dependence when moving from the blue cloud to the

\footnotetext{
${ }^{4}$ Kormendy et al. (2009) also suggest that "extra light ellipticals got their low Sérsic indices by forming in relatively few binary mergers, whereas giant ellipticals have $n>4$ because they formed in larger numbers of mergers of more galaxies at once plus later heating during hierarchical clustering." Interestingly, these authors also find that core Es contain X-ray-emitting gas whereas extra light Es generally do not.
}

red sequence, such that at a given luminosity, quenched, red sequence galaxies are slightly larger. In addition, it is interesting to note that late type galaxies in Figure 4 do not show as strong a trend with concentration as elliptical galaxies, possibly indicative of fewer mergers in their formation history, or perhaps of more minor mergers.

The environmental dependence of the trends just noted are explored in Figure 5 which shows the luminosity versus size relation for blue cloud (top) and red sequence (bottom) elliptical galaxies in low (left) and high density (right) regions keyed to central concentration. Blue cloud elliptical galaxies are found in both low and high density regions, though there are very few small, low luminosity ellipticals in rich environments. We find that blue cloud elliptical galaxies exhibit significantly different slopes than do red sequence galaxies. In addition, red sequence elliptical galaxies exhibit very little change in moving from low to high density regimes, unlike blue cloud galaxies which exhibit a shallower luminosity versus size relation than the general elliptical population. Given the small numbers of blue elliptical galaxies present in our sample $(<200)$, they do not influence the overall luminosity size relation of elliptical galaxies. However at higher redshifts, the blue elliptical population may be significant in both low and high density regimes and may cause a shift in the overall luminosity size relation. Any high redshift study which identifies ellipticals by using the red sequence technique or color cuts (Trujillo et al. 2004, 2006) is likely to miss this population of galaxies.

In summary, Figure 4 and Figure 5 appears to show that variations in concentration (i.e. homology) as well as variations in color (i.e. age) combine to define the scatter about the luminosity-size relationship. The visual impression from Figures 4 and 5, backed up by the results summarized in Tables 1 and 2 , is that variations in homology are manifested in small 
changes the slopes and intercepts of the fiducial luminositysize relation for early-type galaxies. On the other hand, comparing the variation as a function of color in the range spanned by galaxies at various Hubble types suggests that color (i.e. age) plays the defining role here. For example, it is seen that for early-type galaxies the reddest systems tend to be largest and most luminous, and that a related trend appears to hold for late type galaxies, few of which are both red and underluminous. Taken together, these observations seem to provide some evidence for the notion that the origin of the scatter in the luminosity-size relation can be described in the following simple terms: for a given Hubble type, variations in homology change the slope and intercept in the local luminosity-size relationship, while variations in age truncate the size range spanned by galaxies.

\section{IS THE CLUSTER CENTER IMPORTANT?}

In the previous sections we considered the dependence of the luminosity-size relation on global environment and color. Although global environment does influence the mass function of individual types, it is not the only useful measure for testing hierarchical formation models. In fact, the position of a galaxy within a cluster may be more important than global density, since different physical processes occur in the outskirts versus the centers of groups or clusters (Dressler 1980). Boylan-Kolchin et al. (2006) find that the merger history of BCGs, where mergers preferentially occur on radial orbits, would lead to a steeper size-luminosity relation compared to field galaxies. In addition, satellite galaxies could lose their extended gas haloes (starvation/strangulation, e.g. Larson et al. (1980)) by interaction with the intracluster medium and ram pressure stripping (Gunn \& Gott 1972). These two processes are often cited as being important to the creation of S0 galaxies in clusters, though it is hardly an exhaustive list, and a number of other processes could be responsible for the requisite gas loss (e.g. there is evidence that an AGN might be responsible for turning NGC3115 into an S0, c.f. van den Bergh (2009)).

Recent studies with the SDSS have found conflicting results with respect to size-luminosity relationship of BCGs. Lauer et al. (2007), Bernardi et al. (2007), Liu et al. (2008), Coenda \& Muriel (2009) and Bernardi (2009), using the C4 cluster catalog (Miller et al. 2005) and/or the MaxBCG cluster catalog (Koester et al. 2007), find that early-type BCG galaxies are larger at fixed luminosities and show a steeper size-luminosity ( $\mathrm{Y}$ versus $\mathrm{X}$ ) relation than satellite or field galaxies. On the other hand, Guo et al. (2009) and Weinmann et al. (2009) using the Yang et al. (2007) halo catalog find no difference between central and satellite galaxies in the size-luminosity plane.

Figure 6 shows the luminosity-size relation for elliptical galaxies in our sample for field galaxies (in green), satellite galaxies in groups with 10 or more members (in yellow) and BCG galaxies in groups with ten or more members (in orange). Best fit lines for each sample are over-plotted. Points with error bars indicate the 25 th and 75 th quantiles in various size bins. We find that BCG galaxies have a shallower size vs luminosity slope (steeper luminosity vs size slope) consistent with Bernardi (2009) and others. This result may be due to some mild curvature in the luminosity-size relationship, since the BCGs span such a limited range of luminosities (which is why, of course, they have been used as distance indicators), and in any case the slope is difficult to define with confidence because of the small luminosity range spanned. However, it is interesting to note that quantile distribution of luminosities (blue and red error bars) is similar for field and BCG galaxies, where the samples overlap, especially at higher luminosities, implying little difference between BCGs and field galaxy scaling relations in the region of overlap. BCGs show a tighter luminosity size relation than the general elliptical galaxy population with a dispersion of only 0.25 mag. Satellite cluster galaxies show a slightly larger dispersion while field galaxies show the largest dispersion $(0.39 \mathrm{mag})$. This is consistent with previous studies by Bernardi et al. (2007) and Bernardi (2009). These authors suggest that the small dispersion for BCG galaxies indicates that these systems are older (probably the supermassive compact galaxies seen at high redshifts) and have less recent star formation than either satellite or field elliptical galaxies, with growth in size and mass predominantly due to dry minor mergers (Bernardi 2009; Boylan-Kolchin et al. 2006) If the progenitors of these BCGs are dwarfs, then it seems a challenge to understand how metal-rich populations can be built up through the successive mergers of metal poor populations.

There are some additional caveats to bear in mind when comparing Figure 6 with previous work. First we do not probe very massive clusters. Only 731 galaxies are in halos with masses greater than $\log \left(M_{\text {halo }} / M_{\odot}\right)>14.0$. Thus we may be missing the very massive BCG galaxies present in the $\mathrm{C} 4$ or MaxBCG catalogs. Our redshift restrictions $(\mathrm{z}<0.1)$ may also cause us to miss more massive BCGs. Secondly, we have not accounted for sky over-estimates which adversely affect size measurements of massive clustered galaxies. It should be noted that Guo et al. (2009) fit their sample of galaxies with GALFIT (Peng et al.2002) to account for sky over-estimation while Weinmann et al. (2009) did not but found consistent results. We performed a test of our results using the corrections for de Vaucouleurs sizes and magnitudes prescribed by Hyde \& Bernardi (2008). Although the distribution for all ellipticals shifts to larger sizes and brighter magnitudes, the trends remain the same. Thirdly, there may be a discrepancy in the cluster detection algorithm and BCG identification. The $\mathrm{C} 4$ and MaxBCG catalogs use the red-sequence technique to initially identify clusters whereas the Yang group catalog uses a modified friends-of-friends algorithm. The C4 and MaxBCG clusters will therefore miss blue clusters. In addition, recent work by von der Linden et al. (2007) has actually found that not all galaxies identified as central galaxies in the $\mathrm{C} 4$ catalog are centrals. This effect cannot be ruled out for the Yang et al. (2007) group catalog.

Given these many caveats, we conclude that we have relatively little to say about the role played by BCG galaxies in conditioning the luminosity-size relationships explored in this paper. However, they clearly have a part to play, and the presence of BCGs probably goes a long way toward explaining the large sizes of early-type galaxies at the 90th percentile (the final column) in Table 11. We can confirm the tightness of the luminosity size relation of BCGs in comparison to satellite or field ellipticals. We also find no difference between late stage satellite and field galaxies.

\section{WHICH SIZE IS APPROPRIATE?}

Giuricin et al. (1988) first drew attention to the surprising fact that the luminosity-radius relation provides the tightest of all correlations observed among the photometric parameters that can be used to characterize galaxies. This conclusion was subsequently strengthened and confirmed by Gavazzi et al. (1996). More recently van den Bergh (2008) has pointed 


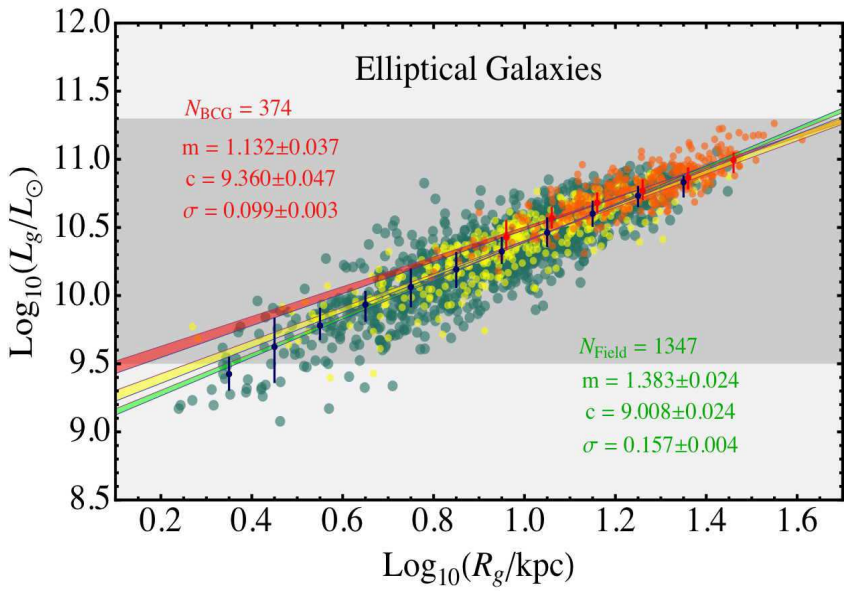

FIG. 6. - g-band luminosity versus g-band Petrosian $\mathrm{R}(90)$ radius in $\mathrm{kpc}$ for $\mathrm{E}$ and $\mathrm{E} / \mathrm{S} 0$ galaxies showing the brightest cluster galaxies ( $\mathrm{BCG}$; orange points), satellite galaxies (yellow points) and field galaxies (green points). Best fit lines for each environments are shown in red, yellow and green. The red (green) lines show the range spanned by the first and third quantile in each luminosity bin.

out that the tightness of the luminosity-radius relation among early-type galaxies is difficult to reconcile with scenarios in which such galaxies are formed via chaotic hierarchical mergers. In the present work we have used the Petrosian (1976) outer radii $\mathrm{R}(90)$ of galaxies that enclose $90 \%$ of the galaxian flux. Similarly van den Bergh (2008) used the radii of galaxies (corrected for reddening) at the faint outer isophote having $\mathrm{B}=25.0 \mathrm{mag} \operatorname{arcsec}^{-2}$. We now inquire whether a similarly tight correlation is observed between the luminosities of galaxies and their effective Petrosian radii $\mathrm{R}(50)$. The relevant observations are collected in Table 3 . The data in this table show strong correlations between galaxy luminosity and half-light radius. However, in all cases, the dispersion in the relations using $\mathrm{R}(50)$ is seen to be slightly larger than that which employs $\mathrm{R}(90)$. Perhaps more surprising is the lack of dependence of the slope of the luminosity-size relation on the morphology of early type galaxies (E, S0 and Sa galaxies) with $\mathrm{R}(90)$ whereas $\mathrm{R}(50)$ does distinguish between different early type galaxies.

We repeated our analysis with $\mathrm{R}(50)$, corrected as specified by Graham et al. (2005) to recover the effective radii and total luminosity. Results are shown in Table 4 and are consistent with those found with $\mathrm{R}(50)$ and $\mathrm{R}(90)$. From Table 5, using the SDSS provided de Vaucouleurs radii (corrected as specified by Hyde \& Bernardi (2008)) leads to a shallower slope but a larger scatter than $\mathrm{R}(90)$ for early type galaxies. The trends remain consistent. In other words the outermost radii of galaxies correlates most strongly with luminosity. This result suggests that factors such as mergers and star formation history affect the internal distribution of galaxy light more strongly than they do the overall size of these objects (see Appendix A).

\section{DISCUSSION}

One of the most unexpected conclusions of the present work is that elliptical galaxies (in all environments) exhibit such a tight correlation between their sizes and their luminosities. This result is unexpected if, as proposed by Toomre (1977), elliptical galaxies all formed from mergers of spirals. Such mergers would be expected to be chaotic and are therefore expected to feed into a wide range of final states. The fact that E galaxies are actually observed to exhibit a tight corre- lation between their sizes and their luminosities suggests that they actually arrived at their present state along rather similar evolutionary tracks. In other words it appears as if ellipticals have both simple morphologies and similar evolutionary histories. This conclusion was also reached by Totani (2009), who noted that "the evolution of early type galaxies must occur ubiquitously, rather than stochastically."

Recent N-body simulations and semi-analytic models (Nipoti et al. 2009b) are able to account for the small scatter in the Fundamental Plane specifically by forcing the formation of $\sim 50 \%$ of the mass of present elliptical galaxies at $\mathrm{z}>1$ and allowing subsequent growth via dry mergers so as to account for the tight spread in ages observed locally Thomas et al. (2005); Graves et al. (2009a b). Simulations have shown that once the FP is established it is relatively insensitive to (a few) episodes of dry major mergers. However the projections of the scaling relations, especially the scatter, are still expected to depend on merger history, orientation, and mass ratios of the dry merger Boylan-Kolchin et al. (2006); Robertson et al. (2006a). Contrary to our results, this should be dependent on environment as there is definite growth of elliptical galaxies occurring in dense environments.

The tight luminosity-size relationship further complicates the now well-known problem of explaining the 'red nuggets' seen at high redshift (highly compact early-type galaxies). The relevant observations are well described in van Dokkum et al. (2008), Toft et al. (2009), Damianov et al. (2008), Collins et al. (2009) and van Dokkum et al. (2009). The central difficulty is that the high-redshift systems are anomalously compact for their masses (or luminosities), but major mergers grow galaxies along the fundamental plane, so it is difficult to reconcile the extreme size growth needed with episodes of dry merging, particularly because the galaxies seen are massive to begin with (but see Valentinuzzi et al. (2009)).

Recently, Bernardi (2009) (and others) have suggested that BCGs could be the low redshift equivalent to high redshift red-nuggets. Around $90 \%$ of the mass of these systems have been established by $\mathrm{z} \sim 1$ and the evolution required is a three-fold increase in size from high-redshifts to the present epoch. Simulations predict that it is possible for a series of many minor dry mergers to successfully grow elliptical galaxy sizes while decreasing their central concentration (Naab et al. 2009; Hopkins et al. 2009a c). However, in simulations Nipoti et al. (2009a) have shown that it is impossible to reproduce both the slope and the scatter of the size-mass relation by dry major or minor mergers while accounting for the red nuggets. In addition BCGs exhibit the smallest scatter in the size-luminosity plane out of all early type galaxies even though their sizes are evolving more strongly than normal ellipticals (Bernardi 2009).

Kormendy et al. (2009) certainly makes a strong case for the assumption that the internal structure of early-type galaxies (i.e. the presence or absence of cores) depends on merger history. The curvature in the scaling relation for elliptical galaxies (noted by other authors) due to BCGs could also be a signature of minor mergers (Boylan-Kolchin et al. 2006), although it is worth emphasizing the important caveat that Masjedi et al. (2007) find that major mergers are the dominant mode of growth at $z<0.3$, and that at higher redshifts minor mergers are difficult to detect directly. In any case, it seems a huge challenge to try to explain the build-up of a metal rich giant population through successive minor dry mergers of metal-poor dwarf populations. This mismatch in metallici- 
ties seems to us to be an absolutely fundamental problem with this basic picture, and this difficulty is only compounded by our observation of a very small dispersion in the luminositysize relation for BCGs.

It seems hard to see how any stochastic process could lead to the very tight luminosity-size relationship reported here, so on the whole our results seem to favor the notion that mergers have not greatly affected the luminosity-size relation of elliptical galaxies. Since mergers are clearly happening (Masjedi et al. 2007), but they do not appear to be having a great effect on the luminosity-size relationship, perhaps the mergers are only superficial signatures masking some more important underlying process (rearranging the deck chairs on the sinking Titanic). In any case, our observations suggest that concentration and age are responsible for much of the variance in the luminosity-size relation, and perhaps this provides a useful constraint on models.

In spite of de-emphasizing the role of mergers in the previous paragraph, it would be a mistake to conclude from our work that the luminosity-size relationship is independent of environment. In fact, a striking feature of Figure 3 is that large elliptical galaxies strongly prefer dense environments. This point is seen particularly clearly by inspection of the range in sizes spanned by the $10 \%$ to $90 \%$ size quantiles tabulated in Table 1 We have already noted that much of this effect may find its origin in the presence of BCGs in our sample, and the physics of size growth in these objects may well differ from that of elliptical galaxies in sparse environments. At the same time, the absence of under-luminous galaxies in dense regions is probably the natural extension of the effect seen by Coenda \& Muriel (2009) in their cluster sample, and follows the general trends noted by Blanton et al. (2003, 2005a) and Hogg et al. (2004), although these authors did not study the morphologies of the galaxies in any detail. The central point emerging from our study is that even though the linear fits to the luminosity-size data for $\mathrm{E}$ is unchanged with environment, galaxies with sizes greater than $\log \left(R_{90} / \mathrm{kpc}\right)>1.2$ are abundant in dense regions while being essentially absent from sparse regions in the field. So even though the form of the luminosity-size relationship seems independent of environment, the range spanned by galaxies described by the relation seems to be a strong function of environment. This result would seem to follow naturally from the work of Zucca et al. (2009), who find, as we also do, that the characteristic magnitude of galaxies is fainter in under-dense regions than it is in over-dense regions.

It is interesting to note that our analysis includes 255 earlytype galaxies in the blue cloud. While this is only a small fraction $(8 \%)$ of the total early-type sample, these are exceptionally interesting objects. Careful re-inspection of the images suggests that some small fraction of these could be misclassifications of later-type galaxies, but the great majority of the classifications seem secure (see Nair \& Abraham (2010)). These blue cloud early types could be ellipticals which have undergone a wet major merger recently, which has led to the reformation of a disk (Wei et al. 2009), although it is interesting to note that blue cloud early types show a shallow slope on the luminosity-size relationship diagram and hence do not behave like disk galaxies. It is not clear how to reconcile this shallower slope with the claim by Wei et al. (2009) that the presence of E and SO galaxies on the blue cloud suggests that wet major merger processes could lead to the reformation of a disk. (The dispersion for these systems are larger and environmentally dependent.) Perhaps wet mergers do not affect the luminosity-size relationship in the same way as dry mergers affect the relationship.

The absence of luminous (or large) galaxies in sparse regions is also notable amongst the late-type population. Figure 3 shows a striking deficiency of faint late-type spirals in high-density regions. Very few Sb or Sc spirals, in regions of above-average density, have $\log L g / L_{\odot}<9.5$. In other words the dispersion in the luminosity-radius relation of galaxies increases steeply towards later morphological types. In the case of spirals of type Sc this increased dispersion is, at least in part, due to a strong population of faint objects that fall below the line defined by the fiducial relationships shown in Table 1 (in other words, the dispersion is skewed to small systems).

Figure 2 and Table 1 also provide some insight into the relationship between elliptical galaxies and S0 systems. The basic conclusion from van den Bergh (2004) that lenticular galaxies are (as a class) less luminous that either elliptical galaxies or spirals of type Sa is strongly reinforced by these data. Furthermore, for S0 galaxies, the dispersion of the data around the fiducial relationship is found to be 0.16 dex. This value is greater than that for Sa galaxies but only at the $1 \sigma$ level, although it is significantly larger than that for elliptical galaxies (over $5 \sigma$ significant). These results seem consistent with the conclusion by van den Bergh (2009) that S0 galaxies are not intermediate between E and Sa galaxies as Hubble (1936) had proposed. Inspection of the data plotted in Figure 2 suggests that the increased dispersion of S0 galaxies is due to the addition of a population of objects that are either exceptionally luminous for their radius, or exceptionally small for their luminosity.

\section{CONCLUSIONS}

The present investigation is based on a sample of 12150 galaxies that are contained in the Sloan Digital Sky Survey (York et al. 2000). Visual morphological classifications by Nair \& Abraham (2010), and environmental information from Blanton et al. (2005b), Baldry et al. (2006) and Yang et al. (2007) were employed to study the dependence of the luminosity-radius relation of these objects on both their morphological type and environmental density. The main conclusions of this work are:

1. The luminosity-size relation of elliptical galaxies is well represented by a simple power law. The scatter about this relationship is very small: for elliptical galaxies, the dispersion is only $0.14 \mathrm{dex}(0.35 \mathrm{mag})$, making this relationship as tight as that defined by the fundamental plane, even though it is based on purely photometric parameters.

2. The scatter about this luminosity-size relation is due to variations in both galaxy central concentration and star-formation history. Increasing central concentration has the effect of making modest changes in the slopes and intercepts of the fiducial relations. Although for all subsets of early-type galaxies the scatter remains small, the scatter in the luminosity-size relationship is $\sim 50 \%$ larger for a small fraction $(<10 \%)$ of early-type galaxies in the blue cloud than on the red sequence.

3. The slope and dispersion about the luminosity-size relationship increases as one proceeds along the Hubble sequence $E \Rightarrow \mathrm{Sa} \Rightarrow \mathrm{Sb} \Rightarrow \mathrm{Sc}$.

4. Elliptical galaxies are presumed to have formed by the chaotic mergers of either smaller ellipticals or of disk- 
like ancestral objects, but it is difficult to see how such a process could result in a very tight luminosity-size relationship.

5. For galaxies of a given Hubble type, the slope of the luminosity-size relationship seems independent of environment. However the range spanned by the luminosities (or sizes) of that class of galaxy seems to be a strong function of environment.

6. The de Vaucouleurs radius, Rdev, and Petrosian half light radius, $\mathrm{R}(50)$, are more sensitive to changes in central concentration and hence merger history than $\mathrm{R}(90)$. Thus it appears mergers only influence the internal structure of galaxies and not their global luminositysize relation.

It is not clear why objects that might have assembled in such very different ways, from differing ancestral objects, should have had evolutionary tracks that converged to show small dispersions around simple power-law forms for their luminosity-size relationships. Perhaps elliptical galaxies started out very differently from each other at $z \sim 3$, but their morphologies converged to similar configurations as they expanded between redshifts $z=3$ and $z=0$ (Johansson et al. 2009).

\section{Acknowledgments}

PN and RGA thank Ivan Baldry for a helpful discussion about possible selection effects affecting the luminosity- radius relations for galaxies in differing environments. PN would also like to thank Gianni Zamorani, Luca Ciotti and Carlo Nipoti for helpful discussions. SvdB would like to thank Leo Girardi, David Hogg, Igor Karachentsev, and John Kormendy for helpful exchanges of e-mails.

Funding for the SDSS and SDSS-II has been provided by the Alfred P. Sloan Foundation, the Participating Institutions, the National Science Foundation, the U.S. Department of Energy, the National Aeronautics and Space Administration, the Japanese Monbukagakusho, the Max Planck Society, and the Higher Education Funding Council for England. The SDSS Web Site is http://www.sdss.org/

The SDSS is managed by the Astrophysical Research Consortium for the Participating Institutions. The Participating Institutions are the American Museum of Natural History, Astrophysical Institute Potsdam, University of Basel, University of Cambridge, Case Western Reserve University, University of Chicago, Drexel University, Fermilab, the Institute for Advanced Study, the Japan Participation Group, Johns Hopkins University, the Joint Institute for Nuclear Astrophysics, the Kavli Institute for Particle Astrophysics and Cosmology, the Korean Scientist Group, the Chinese Academy of Sciences (LAMOST), Los Alamos National Laboratory, the Max-Planck-Institute for Astronomy (MPIA), the MaxPlanck-Institute for Astrophysics (MPA), New Mexico State University, Ohio State University, University of Pittsburgh, University of Portsmouth, Princeton University, the United States Naval Observatory, and the University of Washington.

\section{REFERENCES}

Abraham, R. G., et al. 2007, ApJ, 669, 184

Baldry, I. K., et al. 2006, MNRAS, 373, 469

Baldry, I. K., et al. 2004, ApJ, 600, 681

Bernardi, M. 2009, MNRAS, 395, 1491

Bernardi, M., et al. 2009, eprint arXiv, 0910, 1093

Bernardi, M., et al. 2007, AJ, 133, 1741

Bernardi, M., et al. 2006, AJ, 131, 1288

Bernardi, M., et al. 2003, AJ, 125, 1866

Blanton, M. R. \& Berlind, A. A. 2007, ApJ, 664, 791

Blanton, M. R., et al. 2005a, ApJ, 629, 143

Blanton, M. R., et al. 2005b, AJ, 129, 2562

Blanton, M. R., et al. 2003, ApJ, 592, 819

Bournaud, F., Combes, F., \& Jog, C. J. 2004, A\&A, 418, L27

Boylan-Kolchin, M., Ma, C.-P., \& Quataert, E. 2006, MNRAS, 369, 1081

Cassata, P., et al. 2009, eprint arXiv, 0911, 1158

Ciotti, L., Lanzoni, B., \& Volonteri, M. 2007, ApJ, 658, 65

Coenda, V. \& Muriel, H. 2009, eprint arXiv, 0906, 1939

Collins, C. A., et al. 2009, Nature, 458, 603

Covington, M.,et al. 2008, MNRAS, 384, 94

Dalcanton, J. J., Spergel, D. N., \& Summers, F. J. 1997, ApJv.482, 482, 659

Damjanov, I., et al. 2008, eprint arXiv, 0807, 1744

de Vaucouleurs, G., et al. 1991, Volume 1-3

Deng, X.-F.,et al. 2009, PASP, 121, 683

Desroches, L.-B., et al. 2007, MNRAS, 377, 402

Disney, M. J., et al. 2008, Nature, 455, 1082

Dressler, A. 1980, ApJ, 236, 351

Fukugita, M., et al. 2007, AJ, 134, 579

Gallazzi, A., et al. 2006, MNRAS, 370, 1106

Gavazzi, G., Pierini, D., \& Boselli, A. 1996, A\&A, 312, 397

Girardi, M., et al. 1991, ApJ, 366, 393

Giuricin, G., Mardirossian, F., \& Mezzetti, M. 1988, A\&AS(ISSN 03650138), 72, 151

Graves, G. J., Faber, S. M., \& Schiavon, R. P. 2009a, ApJ, 693, 486

-. 2009b, ApJ, 698, 1590

Graham, A. W., et al. 2005, AJ, 130, 1535

Gunn, J. E. \& Gott, J. R. 1972, ApJ, 176, 1

Guo, Y., et al. 2009, eprint arXiv, 0901, 1150

Hogg, D. W., et al. 2004, ApJ, 601, L29

Hopkins, P. F., et al. 2009a, eprint arXiv, 0909, 2039
Hopkins, P. F., et al. 2009b, MNRAS, 1043

Hopkins, P. F., et al. 2009c, ApJ, 691, 1424

Holmberg, E. 1958, Lund Medd. Astron. Obs. Ser. II, 136, 1

Hubble, E. P. 1936, Realm of the Nebulae

Hyde, J. B. \& Bernardi, M. 2008, eprint arXiv, 0810, 4922

Johansson, P. H., Naab, T., \& Ostriker, J. P. 2009, ApJ, 697, L38

Kauffmann, G., et al. 2003a, MNRAS, 341, 33

Kauffmann, G., et al. 2003b, MNRAS, 341, 54

Koester, B. P., et al. 2007, ApJ, 660, 239

Kormendy, J., et al. 2009, ApJS, 182, 216

Larson, R. B., Tinsley, B. M., \& Caldwell, C. N. 1980, ApJ, 237, 692

Lauer, T. R., et al. 2007, ApJ, 662, 808

Liu, F. S., et al. 2008, MNRAS, 385, 23

Masjedi, M., Hogg, D. W., \& Blanton, M. R. 2008, ApJ, 679, 260

Miller, C. J., et al. 2005, AJ, 130, 968

Mo, H. J. \& White, S. D. M. 1996, MNRAS, 282, 347

Naab, T., Johansson, P. H., \& Ostriker, J. P. 2009, eprint arXiv, 0903, 1636

Nair, P. B. \& Abraham, R. G. 2010, ApJS, 186, 427

Nipoti, C., Treu, T., Auger, M. W., \& Bolton, A. S. 2009a, ApJ, 706, L86

Nipoti, C., Treu, T., \& Bolton, A. S. 2009b, ApJ, 703, 1531

Park, C., Gott, J. R., \& Choi, Y.-Y. 2008, ApJ, 674, 784

Peng, C. Y., Ho, L. C., Impey, C. D., \& Rix, H.-W. 2002, AJ, 124, 266

Robertson, B., et al. 2006a, ApJ, 645, 986

Robertson, B., et al. 2006b, ApJ, 641, 21

Sandage, A. \& Bedke, J. 1994, Washington

Sandage, A. \& Tammann, G. A. 1981, Washington: Carnegie Institution

Schlegel, D. J., Finkbeiner, D. P., \& Davis, M. 1998, ApJ, 500, 525

Shankar, F., \& Bernardi, M. 2009, MNRAS, 396, L76

Shen, S., et al. 2003, MNRAS, 343, 978

Strauss, M. A., et al. 2002, AJ, 124, 1810

Thomas, D., et al. 2005, ApJ, 621, 673

Toft, S., et al. 2009, eprint arXiv, 0909, 0750

Toomre, A. 1977, In: ARA\&A, (A78-16576 04-90) Palo Alto, 15, 437

Totani, T. 2009, eprint arXiv, 0908, 3295

Trujillo, I., et al. 2009, eprint arXiv, 0901, 1032

Trujillo, I., et al. 2006, ApJ, 650, 18

Trujillo, I., et al. 2004, ApJ, 604, 521

Valentinuzzi, T., et al. 2009, ApJ, 712, 226 
van den Bergh, S. 1998, Galaxy morphology and classification, Cambridge ;

New York : Cambridge University Press

-. 2004, ApJ, 601, L37

-. 2008, A\&A, 490, 97

-. 2009, ApJ, 702, 1502

van Dokkum, P. G., Kriek, M., \& Franx, M. 2009, Nature, 460, 717

van Dokkum, P., et al. 2008, arXiv, 0802, 4094

von der Linden, A., et al. 2007, MNRAS, 379, 867
Wei, L. H., et al. 2010, ApJ, 708, 841

Weinmann, S. M., et al. 2009, MNRAS, 394, 1213

Yang, X., et al. 2007, ApJ, 671, 153

York, D. G., et al. 2000, AJ, 120, 1579

Zucca, E., et al. 2009, eprint arXiv, 0909, 4674

APPENDIX

A. BIASES IN MEASUREMENTS OF SIZE?

Three issues need to be kept in mind while using SDSS sizes and luminosities:

1. SDSS has a known problem of sky over-estimation near bright galaxies especially in dense regions. We have partially compensated for this by selecting a clean sample of galaxies with no overlapping companions. In addition, we ran our analysis excluding objects with $r$-band model magnitudes $r<14$ where the effects of sky overestimation are severe. Changes in the fits are small and within the error bars quoted. Our overall results and conclusions remain unaffected.

\section{Petrosian sizes have not been corrected for seeing.}

Hyde \& Bernardi (2008) use the SDSS seeing corrected de Vaucouleurs size (RdeV) and magnitude as they find the SDSS Petrosian quantities (which are not seeing corrected) show a slight bias where higher redshift early type galaxies are larger than lower redshift early type galaxies at similar luminosities. As we probe a smaller redshift range $\mathrm{z}<0.1$ than Hyde et al. $(\mathrm{z}<0.3)$, we do not expect seeing to be important in measures of R(90). To illustrate this, Figure 7 (a) shows the median g-band Petrosian $\mathrm{R}(90)$ size in bins of luminosity for ellipticals galaxies. The error bars show the 25 th and 75 th quantiles. The blue points represent galaxies below a redshift of 0.05 while the red points show galaxies above $\mathrm{z}$ of 0.05 . We find galaxies in the higher redshift bin are slightly larger at any given luminosity which could imply a seeing bias. However, Figure 7(b) shows the median stellar mass in bins of luminosity where we find a comparable redshift dependence of mass on luminosity. This is because the higher redshift bin has more massive galaxies (volume effect) and fewer lower mass systems due to our apparent magnitude cut $(\mathrm{g}<16)$. Thus the offset of size measures at similar luminosities is because of different mass selections and not due to seeing. Surprisingly Figure 7(c) shows the Petrosian R(50) size has a comparable redshift dependent offset with luminosity. Figure 7 (d) shows the g-band de Vaucouleurs size (Rdev) versus luminosity, both corrected for over-estimation of sky as specified by Hyde \& Bernardi (2008). The redshift dependent offset in the size measures are smaller but the scatter in each redshift bin is larger than the scatter in $\mathrm{R}(90)$ or $\mathrm{R}(50)$. This is especially noticeable in the highest luminosity bins where the scatter in $\mathrm{R}(90)$ is much smaller than Rdev.

3. The choice of a size metric influences the scaling relations.

Figure 8 shows the dependence of Petrosian R(90) (top) and de Vaucouleurs radii (bottom) on luminosity (left) and stellar mass (right) as a function of concentration. The most concentrated galaxies are shown in red $(\mathrm{C}>0.5)$, followed by green $(0.4<\mathrm{C}<0.5)$, with the least concentrated galaxies shown in blue $(\mathrm{C}<0.4)$. The de Vaucouleurs measure of size is strongly dependent on the concentration of the galaxy, especially at high masses/luminosities whereas the dependence of R(90) on concentration is mild. Thus Rdev is more sensitive to the internal structure of early type galaxies. The curvature seen in high density environments may be related to physical processes which change the central concentration of galaxies while not changing $\mathrm{R}(90)$. In other words, the reason for differing results among various authors regarding curvature in scaling relations may not be because of seeing dependent measures of size but on the effect of concentration on those measures. Thus we would recommend using R(90) which is relatively insensitive to both seeing and central concentration to the de Vaucouleurs size measure. It should be noted that our results for elliptical galaxies do not change when using Rdev. Table 5 shows fits for elliptical galaxies using de Vaucouleurs size and magnitude (sky corrected). We find the slopes are the same in low and high density environments with a smaller scatter in high density environments. BCG galaxies in clusters show a shallower luminosity-size relation (steeper size-luminosity relation) than satellite galaxies.

\section{B. COMPARISON OF ENVIRONMENT ESTIMATES}

Figure 9 shows the g-band luminosity of Sc galaxies versus the g-band Petrosian R(90) radius in low and high density regions keyed to the color of the galaxy using the three environmental measures available. The first panel shows the distribution of $\mathrm{Sc}$ galaxies in low and high density regions as defined by Blanton et al. (2005b), the second row as defined by Yang et al. (2007) and the third row as defined by Baldry et al. (2006). The last row is defined using both Yang and Baldry measures of environment. The black line in each panel shows the relation for elliptical galaxies. This figure shows that many small Sc galaxies in clusters are missed when using the Blanton et al. measure of environment, which leads to a spurious apparent evolution of the $\mathrm{L}$ vs $\mathrm{R}$ relation for Sc galaxies from the field to cluster. This bias is largest for late type galaxies and smallest for elliptical galaxies. We thus restrict ourselves to the Baldry and Yang measures of environment, compared in Figure 10 . We find for $\mathrm{N}<3$, some galaxies are in over-dense regions implying satellites or companions were missed but most are in under-dense regions (i.e. field galaxies). 

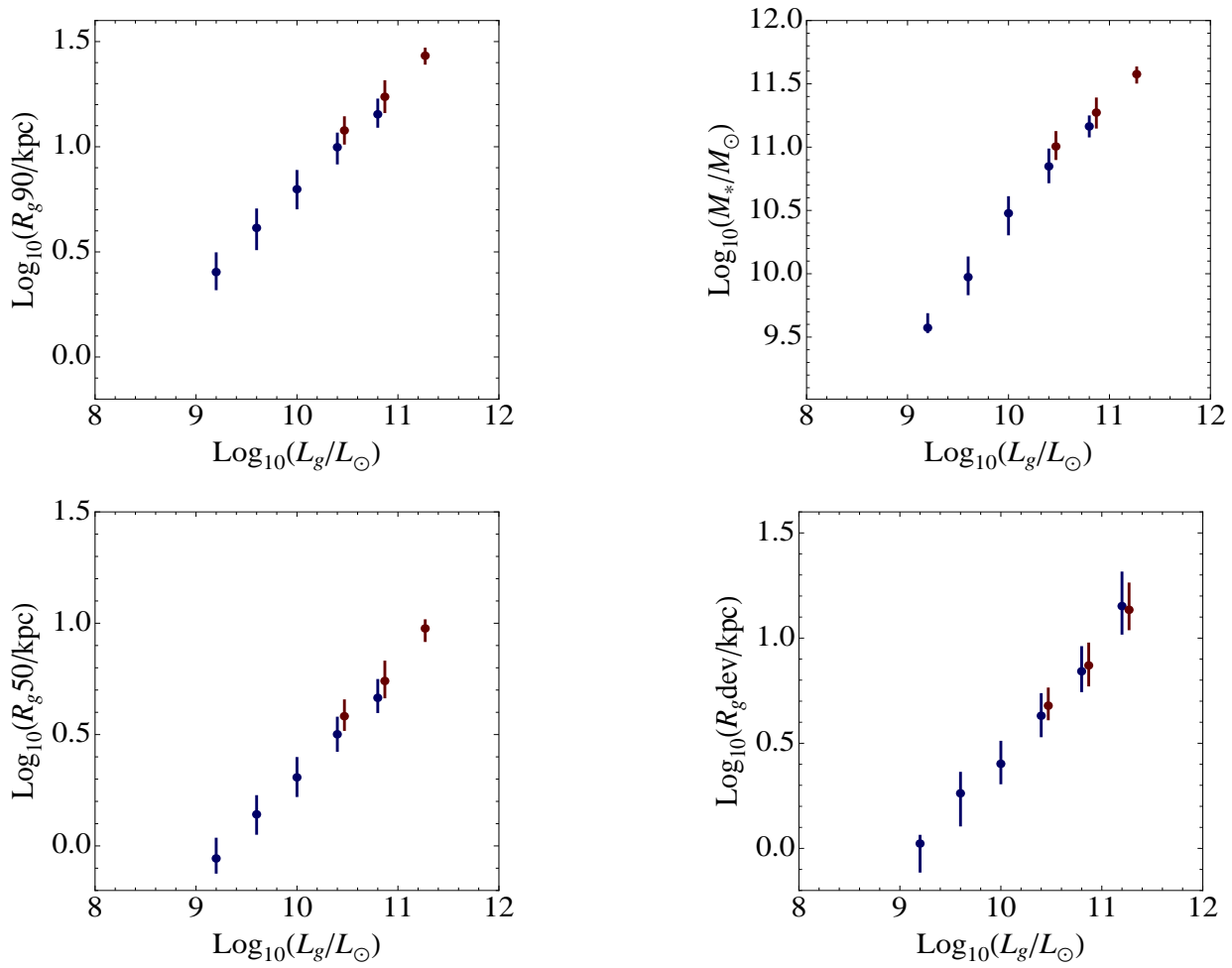

FIG. 7.- (a) g-band Petrosian R(90) radius in kpc versus g-band luminosity for ellipitical galaxies, (b) stellar mass versus g-band luminosity for ellipitical galaxies, (c) g-band Petrosian R(50) radius in kpc versus g-band luminosity for ellipitical galaxies and (d) g-band de Vaucouleurs radius in kpc versus g-band luminosity (corrected for sky subtraction as per Hyde \& Bernardi (2008)) for ellipitical galaxies. The red points show galaxies at redshifts above 0.05 while blue points show galaxies below it. The red points are offset for clarity.
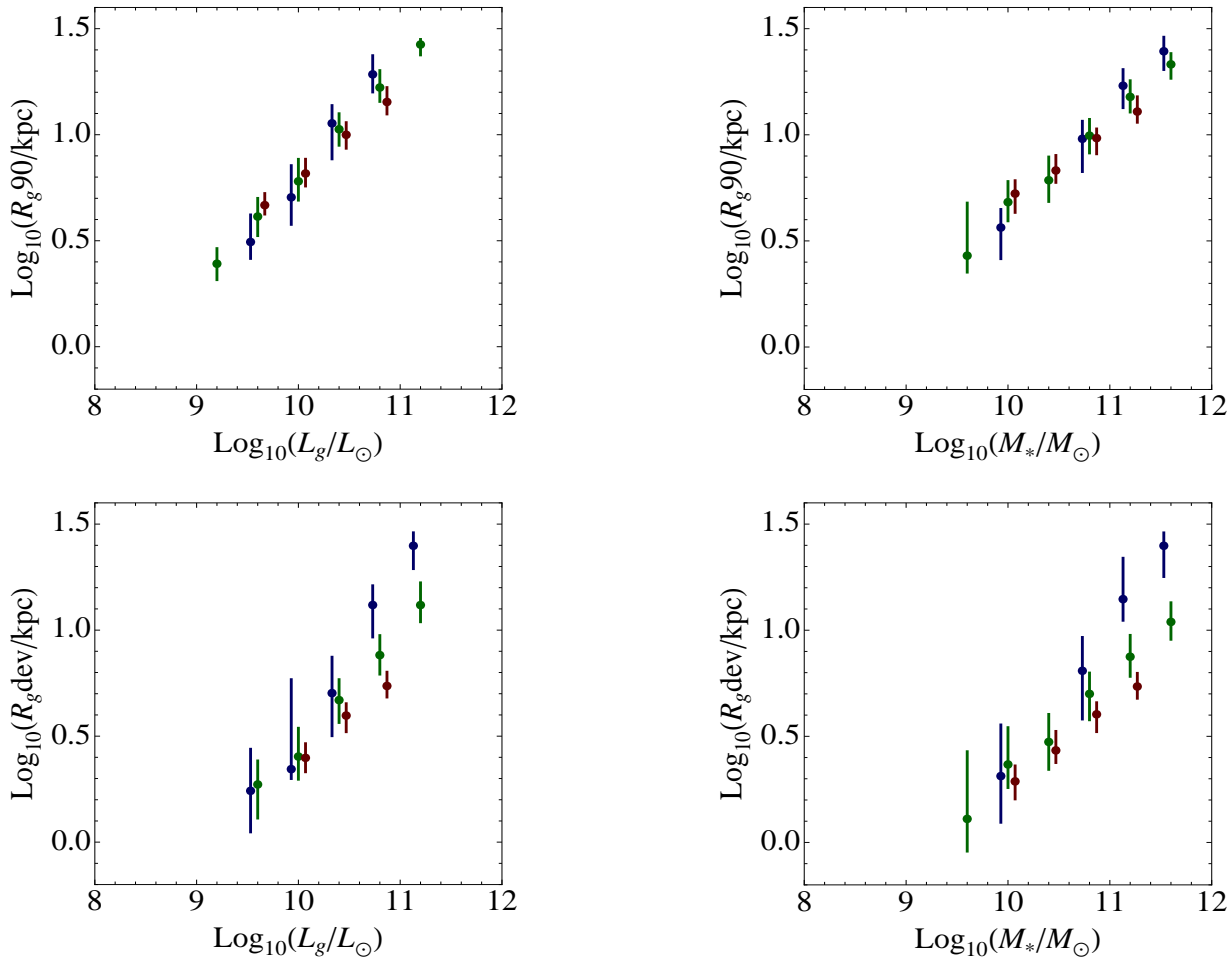

FIG. 8.- Top: g-band Petrosian R(90) radii in kpc versus (a) luminosity and (b) stellar mass. Bottom: $R_{\text {dev }}$ versus (c) Dev luminosity and (d) stellar mass. Points are keyed to central concentration. The most concentrated galaxies $\mathrm{C}>0.5$ are shown in red, followed by green $0.4<\mathrm{C}<0.5$ with the least concentrated galaxies shown in blue $(\mathrm{C}<0.4)$. de Vaucouleurs measure of size is very dependent on the concentration of the galaxy, especially at high masses/luminosities. The red points are offset for clarity. 

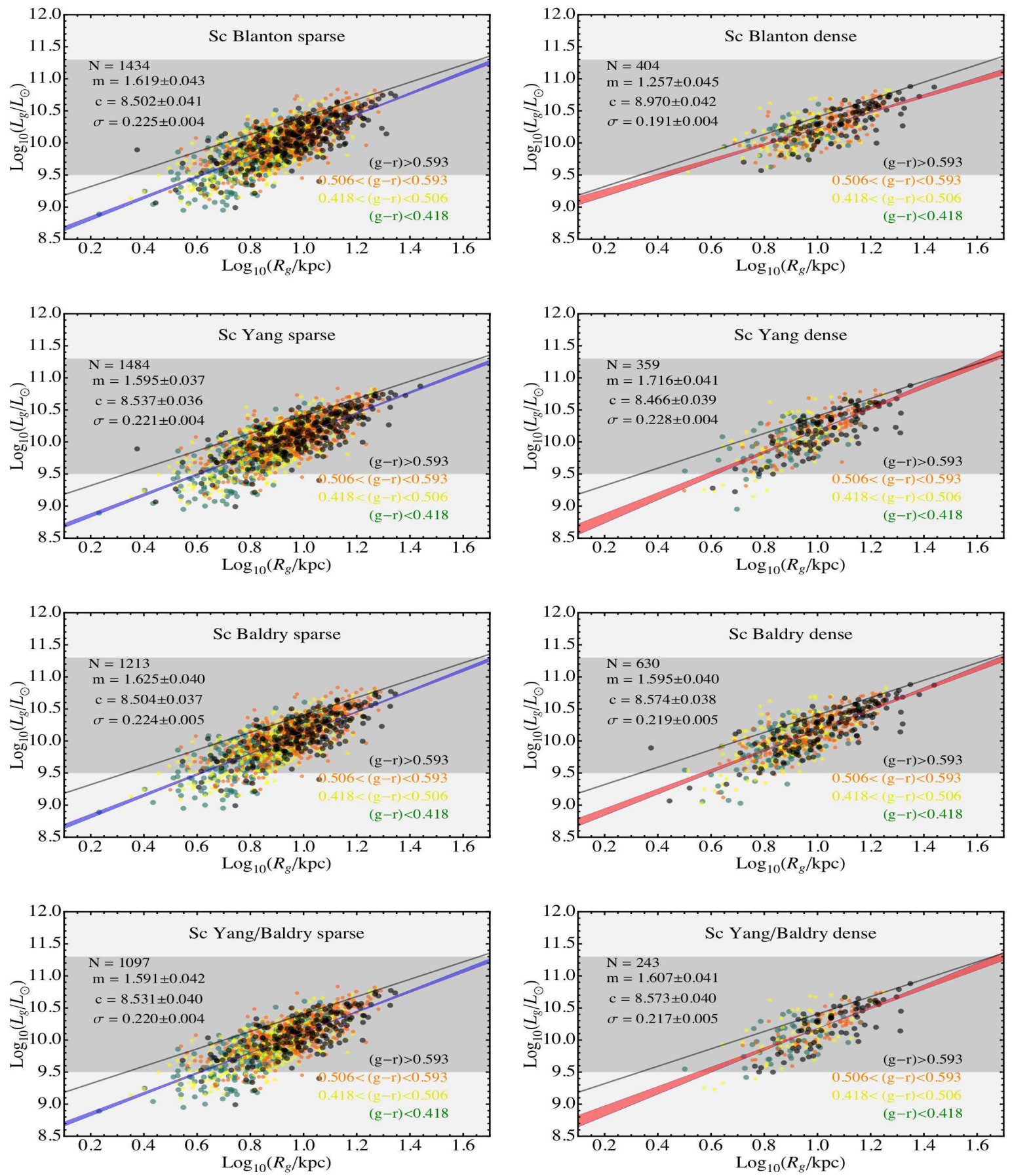

FIG. 9.- g-band Luminosity versus g-band Petrosian R(90) radius in kpc in low (left) and high (right) density environments for Sc galaxies only. The top three rows show environments defined by Blanton et al 2005, Yang et al. 2007 and Baldry et al. 2006. The bottom panel shows L vs. R for galaxies defined using both Yang and Baldry estimates of environmental density. The points are keyed to $g-r$ color quartiles. Black points are the reddest galaxies in each panel, followed by orange, then yellow with green points being the bluest galaxies. 
TABLE 1

LUMINOSITY-SIZE R(90) RELATIONSHIPS

\begin{tabular}{|c|c|c|c|c|c|c|}
\hline Class & $\mathrm{N}$ & $\begin{array}{c}\text { Slope } \\
{\left[\log \left(L_{\odot}\right)\right]}\end{array}$ & $\begin{array}{c}\text { Intercept } \\
{\left[\frac{\log \left(L_{\odot}\right)}{\log (\mathrm{kpc})}\right]}\end{array}$ & $\begin{array}{l}\text { Dispersion } \\
{\left[\log \left(L_{\odot}\right)\right]}\end{array}$ & $\begin{array}{c}\text { - Interquantil } \\
10 \% \text { quantile } \\
{[\log (\mathrm{kpc})]}\end{array}$ & $\begin{array}{l}\text { Size Range - } \\
90 \% \text { quantile } \\
{[\log (\mathrm{kpc})]}\end{array}$ \\
\hline \multicolumn{7}{|c|}{ Elliptical galaxies } \\
\hline All & 3163 & $1.378 \pm 0.014$ & $9.026 \pm 0.016$ & $0.1425 \pm 0.0021$ & 0.65 & 1.36 \\
\hline Sparse & 801 & $1.36 \pm 0.029$ & $9.032 \pm 0.031$ & $0.1619 \pm 0.0046$ & 0.56 & 1.24 \\
\hline Rich & 1514 & $1.319 \pm 0.02$ & $9.101 \pm 0.023$ & $0.1271 \pm 0.0032$ & 0.74 & 1.4 \\
\hline Rich $N \geq 10$ & 766 & $1.316 \pm 0.021$ & $9.108 \pm 0.025$ & $0.1224 \pm 0.0045$ & 0.7 & $\begin{array}{l}1.4 \\
1.43\end{array}$ \\
\hline Rich BCG $N \geq 10$ & 374 & $1.132 \pm 0.038$ & $9.36 \pm 0.048$ & $0.09946 \pm 0.0041$ & 1. & 1.45 \\
\hline Rich satellite $N \geq 10$ & 392 & $1.263 \pm 0.04$ & $9.138 \pm 0.042$ & $0.1346 \pm 0.0066$ & 0.63 & 1.29 \\
\hline Red sequence & 2908 & $1.403 \pm 0.014$ & $8.995 \pm 0.016$ & $0.1357 \pm 0.0023$ & 0.66 & 1.36 \\
\hline Blue cloud & 255 & $1.185 \pm 0.057$ & $9.273 \pm 0.064$ & $0.1896 \pm 0.011$ & 0.55 & 1.31 \\
\hline \multicolumn{7}{|c|}{ So galaxies } \\
\hline All & 1893 & $1.335 \pm 0.018$ & $9.043 \pm 0.017$ & $0.1636 \pm 0.0033$ & 0.56 & 1.19 \\
\hline Sparse & 751 & $1.295 \pm 0.028$ & $9.086 \pm 0.026$ & $0.1597 \pm 0.0043$ & 0.55 & 1.16 \\
\hline Rich & 562 & $1.352 \pm 0.032$ & $9.012 \pm 0.032$ & $0.1665 \pm 0.0055$ & 0.61 & 1.22 \\
\hline Rich $N \geq 10$ & 306 & $1.321 \pm 0.043$ & $9.02 \pm 0.041$ & $0.1588 \pm 0.0067$ & 0.57 & 1.17 \\
\hline Rich BCG $N \geq 10$ & 32 & $0.8086 \pm 0.11$ & $9.678 \pm 0.11$ & $0.09644 \pm 0.013$ & 0.78 & 1.26 \\
\hline Rich satellite $\mathrm{N} \geq 10$ & 274 & $1.272 \pm 0.046$ & $9.048 \pm 0.042$ & $0.157 \pm 0.0067$ & 0.56 & 1.15 \\
\hline Red sequence & 1520 & $1.315 \pm 0.019$ & $9.04 \pm 0.018$ & $0.1539 \pm 0.0031$ & 0.57 & 1.18 \\
\hline Blue cloud & 373 & $1.316 \pm 0.048$ & $9.144 \pm 0.047$ & $0.175 \pm 0.0073$ & 0.54 & 1.21 \\
\hline \multicolumn{7}{|c|}{ Sa galaxies } \\
\hline All & 1955 & $1.337 \pm 0.022$ & $9.008 \pm 0.023$ & $0.1604 \pm 0.0031$ & 0.63 & 1.25 \\
\hline Sparse & 883 & $1.324 \pm 0.033$ & $9.018 \pm 0.033$ & $0.1536 \pm 0.0044$ & 0.63 & 1.23 \\
\hline Rich & 484 & $1.322 \pm 0.04$ & $9.032 \pm 0.042$ & $0.1606 \pm 0.0058$ & 0.65 & 1.27 \\
\hline Rich $N \geq 10$ & 237 & $1.291 \pm 0.059$ & $9.031 \pm 0.06$ & $0.1657 \pm 0.008$ & 0.65 & 1.23 \\
\hline Rich BCG N $>10$ & 22 & $1.167 \pm 0.25$ & $9.278 \pm 0.29$ & $0.1318 \pm 0.016$ & 0.94 & 1.31 \\
\hline Rich satellite $N>10$ & 215 & $1.229 \pm 0.074$ & $9.079 \pm 0.075$ & $0.1648 \pm 0.0075$ & 0.64 & 1.21 \\
\hline Red sequence & 1001 & $1.231 \pm 0.033$ & $9.078 \pm 0.033$ & $0.1619 \pm 0.0048$ & 0.61 & 1.23 \\
\hline Blue cloud & 954 & $1.4 \pm 0.028$ & $8.975 \pm 0.029$ & $0.1513 \pm 0.0039$ & 0.68 & 1.26 \\
\hline \multicolumn{7}{|c|}{ Sb galaxies } \\
\hline All & 2736 & $1.449 \pm 0.022$ & $8.854 \pm 0.024$ & $0.1719 \pm 0.0028$ & 0.73 & 1.28 \\
\hline Sparse & 1281 & $1.424 \pm 0.027$ & $8.876 \pm 0.029$ & $0.1717 \pm 0.0039$ & 0.71 & 1.27 \\
\hline Rich & 615 & $1.502 \pm 0.046$ & $8.812 \pm 0.05$ & $0.1684 \pm 0.0047$ & 0.78 & 1.32 \\
\hline Rich $N \geq 10$ & 264 & $1.479 \pm 0.066$ & $8.8 \pm 0.07$ & $0.1682 \pm 0.0069$ & 0.77 & 1.3 \\
\hline Rich BCG N $\geq 10$ & 36 & $0.7813 \pm 0.14$ & $9.738 \pm 0.16$ & $0.1198 \pm 0.017$ & 0.95 & 1.39 \\
\hline Rich satellite $\mathrm{N}>10$ & 228 & $1.429 \pm 0.077$ & $8.833 \pm 0.08$ & $0.1636 \pm 0.0067$ & 0.76 & 1.25 \\
\hline Red sequence & 788 & $1.399 \pm 0.036$ & $8.835 \pm 0.039$ & $0.1777 \pm 0.0049$ & 0.69 & 1.31 \\
\hline Blue cloud & 1948 & $1.472 \pm 0.025$ & $8.859 \pm 0.026$ & $0.1611 \pm 0.0028$ & 0.74 & 1.27 \\
\hline \multicolumn{7}{|c|}{ Sc galaxies } \\
\hline All & 1843 & $1.63 \pm 0.031$ & $8.514 \pm 0.03$ & $0.2233 \pm 0.0037$ & 0.65 & 1.19 \\
\hline Sparse & 1098 & $1.591 \pm 0.04$ & $8.531 \pm 0.039$ & $0.2203 \pm 0.0049$ & 0.63 & 1.17 \\
\hline Rich & 243 & $1.607 \pm 0.11$ & $8.573 \pm 0.12$ & $0.2168 \pm 0.01$ & 0.76 & 1.22 \\
\hline Rich $N>10$ & 112 & $1.684 \pm 0.14$ & $8.478 \pm 0.15$ & $0.2059 \pm 0.013$ & 0.72 & 1.23 \\
\hline Rich BCG N $>10$ & 8 & $0.637 \pm 0.24$ & $9.854 \pm 0.27$ & $0.08042 \pm 0.021$ & 0.9 & 1.3 \\
\hline Rich satellite $\mathrm{N} \geq 10$ & 104 & $1.636 \pm 0.14$ & $8.509 \pm 0.14$ & $0.2014 \pm 0.015$ & 0.72 & 1.21 \\
\hline Red sequence & 409 & $1.798 \pm 0.092$ & $8.247 \pm 0.092$ & $0.2034 \pm 0.0088$ & 0.7 & 1.21 \\
\hline Blue cloud & 1434 & $1.636 \pm 0.034$ & $8.538 \pm 0.032$ & $0.2194 \pm 0.0039$ & 0.64 & 1.18 \\
\hline
\end{tabular}




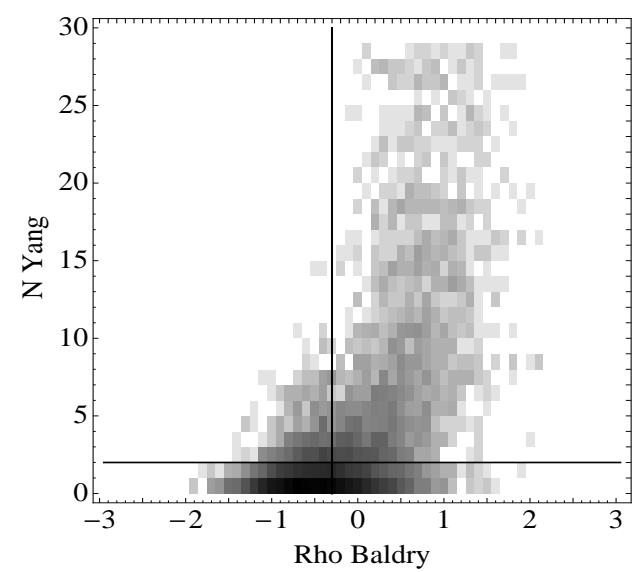

FIG. 10.- Density plot showing comparison of Baldry et al. (2006) nearest neighbor environmental estimate versus Yang et al. (2007) group number estimate. The vertical line show the median Baldry density estimate $(\log \Sigma=-0.32)$ while the horizontal line $(N=2)$ is our demarcation for galaxies in the field versus groups/clusters. $\mathrm{N} \leq 2$ are under dense regions. $\mathrm{N}>2$ are over-dense regions. For clarity, we do not show groups with more than 30 members in this plot.

TABLE 2

LUMINOSITY-SIZE R(90) RELATIONSHIPS

\begin{tabular}{|c|c|c|c|c|c|c|}
\hline Class & $\mathrm{N}$ & $\begin{array}{c}\text { Slope } \\
{\left[\log \left(L_{\odot}\right)\right]}\end{array}$ & $\begin{array}{c}\text { Intercept } \\
{\left[\frac{\log \left(L_{\odot}\right)}{\log (\mathrm{kpc})}\right]}\end{array}$ & $\begin{array}{l}\text { Dispersion } \\
{\left[\log \left(L_{\odot}\right)\right]}\end{array}$ & $\begin{array}{c}\text { - Interquantile } \\
10 \% \text { quantile } \\
{[\log (\mathrm{kpc})]}\end{array}$ & $\begin{array}{c}\text { Size Range - } \\
90 \% \text { quantile } \\
{[\log (\mathrm{kpc})]}\end{array}$ \\
\hline \multicolumn{7}{|c|}{ E galaxies } \\
\hline All & 3163 & $1.378 \pm 0.014$ & $9.026 \pm 0.016$ & $0.1425 \pm 0.0024$ & 0.65 & 1.36 \\
\hline Low Conc Red Sequence & 671 & $1.354 \pm 0.027$ & $9.022 \pm 0.033$ & $0.1641 \pm 0.0057$ & 0.54 & 1.43 \\
\hline High Conc Red Sequence & 743 & $1.52 \pm 0.031$ & $8.885 \pm 0.032$ & $0.1239 \pm 0.0037$ & 0.68 & 1.23 \\
\hline Low Conc Rich & 382 & $1.274 \pm 0.043$ & $9.132 \pm 0.055$ & $0.1509 \pm 0.0089$ & 0.66 & 1.45 \\
\hline High Conc Rich & 328 & $1.405 \pm 0.053$ & $9.016 \pm 0.058$ & $0.1249 \pm 0.0063$ & 0.78 & 1.28 \\
\hline Low Conc Sparse & 213 & $1.24 \pm 0.048$ & $9.153 \pm 0.052$ & $0.1995 \pm 0.012$ & 0.42 & 1.28 \\
\hline High Conc Sparse & 226 & $1.544 \pm 0.065$ & $8.848 \pm 0.063$ & $0.1435 \pm 0.0081$ & 0.63 & 1.14 \\
\hline
\end{tabular}


TABLE 3

LUMINOSITY-SIZE R(50) RELATIONSHIPS

\begin{tabular}{|c|c|c|c|c|c|c|}
\hline Class & $\mathrm{N}$ & $\begin{array}{c}\text { Slope } \\
{\left[\log \left(L_{\odot}\right)\right]}\end{array}$ & $\begin{array}{c}\text { Intercept } \\
{\left[\frac{\log \left(L_{\odot}\right)}{\log (\mathrm{kpc})}\right]}\end{array}$ & $\begin{array}{l}\text { Dispersion } \\
{\left[\log \left(L_{\odot}\right)\right]}\end{array}$ & $\begin{array}{c}\text { - Interquantil } \\
10 \% \text { quantile } \\
{[\log (\mathrm{kpc})]}\end{array}$ & $\begin{array}{c}\text { Size Range - } \\
90 \% \text { quantile } \\
{[\log (\mathrm{kpc})]}\end{array}$ \\
\hline \multicolumn{7}{|c|}{ Elliptical galaxies } \\
\hline All & 3163 & $1.341 \pm 0.015$ & $9.718 \pm 0.0094$ & $0.1556 \pm 0.0026$ & 0.17 & 0.872 \\
\hline Sparse & 801 & $1.325 \pm 0.033$ & $9.708 \pm 0.018$ & $0.1738 \pm 0.0049$ & 0.085 & 0.766 \\
\hline Rich & 1514 & $1.271 \pm 0.018$ & $9.776 \pm 0.013$ & $0.1368 \pm 0.0034$ & 0.26 & 0.921 \\
\hline Rich $N \geq 10$ & 766 & $1.271 \pm 0.021$ & $9.776 \pm 0.016$ & $0.1327 \pm 0.0043$ & 0.23 & 0.963 \\
\hline Rich BCG $N \geq 10$ & 374 & $0.9983 \pm 0.041$ & $10.01 \pm 0.031$ & $0.1089 \pm 0.0042$ & 0.51 & 1. \\
\hline Rich satellite $N \geq 10$ & 392 & $1.265 \pm 0.036$ & $9.755 \pm 0.021$ & $0.1421 \pm 0.0071$ & 0.16 & 0.802 \\
\hline Red sequence & 2908 & $1.37 \pm 0.016$ & $9.699 \pm 0.0098$ & $0.1488 \pm 0.0025$ & 0.18 & 0.877 \\
\hline Blue cloud & 255 & $1.111 \pm 0.061$ & $9.881 \pm 0.037$ & $0.2058 \pm 0.013$ & 0.082 & 0.844 \\
\hline \multicolumn{7}{|c|}{ So galaxies } \\
\hline All & 1893 & $1.17 \pm 0.021$ & $9.723 \pm 0.011$ & $0.1866 \pm 0.003$ & 0.096 & 0.768 \\
\hline Sparse & 751 & $1.154 \pm 0.026$ & $9.736 \pm 0.012$ & $0.1766 \pm 0.0043$ & 0.082 & 0.747 \\
\hline Rich & 562 & $1.184 \pm 0.035$ & $9.702 \pm 0.02$ & $0.1913 \pm 0.0067$ & 0.13 & 0.797 \\
\hline Rich $N \geq 10$ & 306 & $1.156 \pm 0.043$ & $9.687 \pm 0.022$ & $0.1828 \pm 0.0072$ & 0.13 & 0.747 \\
\hline Rich BCG $N \geq 10$ & 32 & $0.677 \pm 0.11$ & $10.13 \pm 0.063$ & $0.1037 \pm 0.014$ & 0.32 & 0.82 \\
\hline Rich satellite $N \geq 10$ & 274 & $1.1 \pm 0.054$ & $9.692 \pm 0.026$ & $0.1795 \pm 0.007$ & 0.098 & 0.741 \\
\hline Red sequence & 1520 & $1.152 \pm 0.024$ & $9.713 \pm 0.012$ & $0.1777 \pm 0.0037$ & 0.096 & 0.753 \\
\hline Blue cloud & 373 & $1.14 \pm 0.05$ & $9.81 \pm 0.029$ & $0.2038 \pm 0.0081$ & 0.093 & 0.815 \\
\hline \multicolumn{7}{|c|}{ Sa galaxies } \\
\hline All & 1955 & $1.101 \pm 0.021$ & $9.685 \pm 0.013$ & $0.1834 \pm 0.0034$ & 0.16 & 0.878 \\
\hline Sparse & 883 & $1.101 \pm 0.034$ & $9.677 \pm 0.021$ & $0.1755 \pm 0.0046$ & 0.16 & 0.86 \\
\hline Rich & 484 & $1.074 \pm 0.035$ & $9.719 \pm 0.024$ & $0.1796 \pm 0.006$ & 0.17 & 0.907 \\
\hline Rich $N \geq 10$ & 237 & $0.995 \pm 0.056$ & $9.724 \pm 0.036$ & $0.1911 \pm 0.0086$ & 0.17 & 0.883 \\
\hline Rich BCG N $>10$ & 22 & $0.8248 \pm 0.18$ & $9.97 \pm 0.15$ & $0.1306 \pm 0.015$ & 0.46 & 0.958 \\
\hline Rich satellite $N>10$ & 215 & $0.94 \pm 0.07$ & $9.742 \pm 0.042$ & $0.192 \pm 0.0092$ & 0.16 & 0.828 \\
\hline Red sequence & 1001 & $0.9785 \pm 0.027$ & $9.732 \pm 0.015$ & $0.1896 \pm 0.0055$ & 0.14 & 0.845 \\
\hline Blue cloud & 954 & $1.219 \pm 0.029$ & $9.628 \pm 0.021$ & $0.1715 \pm 0.0039$ & 0.26 & 0.899 \\
\hline \multicolumn{7}{|c|}{ Sb galaxies } \\
\hline All & 2736 & $1.243 \pm 0.02$ & $9.499 \pm 0.015$ & $0.1826 \pm 0.0028$ & 0.33 & 0.955 \\
\hline Sparse & 1281 & $1.23 \pm 0.03$ & $9.499 \pm 0.022$ & $0.1799 \pm 0.0041$ & 0.33 & 0.935 \\
\hline Rich & 615 & $1.279 \pm 0.04$ & $9.496 \pm 0.032$ & $0.1831 \pm 0.0058$ & 0.36 & 0.977 \\
\hline Rich $N \geq 10$ & 264 & $1.215 \pm 0.063$ & $9.501 \pm 0.047$ & $0.1944 \pm 0.0095$ & 0.33 & 0.952 \\
\hline Rich BCG $N \geq 10$ & 36 & $0.6232 \pm 0.19$ & $10.15 \pm 0.16$ & $0.1265 \pm 0.017$ & 0.54 & 1.04 \\
\hline Rich satellite $\mathrm{N}>10$ & 228 & $1.146 \pm 0.078$ & $9.521 \pm 0.053$ & $0.1875 \pm 0.0094$ & 0.32 & 0.925 \\
\hline Red sequence & 788 & $1.094 \pm 0.035$ & $9.563 \pm 0.025$ & $0.1991 \pm 0.0053$ & 0.24 & 0.97 \\
\hline Blue cloud & 1948 & $1.321 \pm 0.025$ & $9.458 \pm 0.018$ & $0.1722 \pm 0.0029$ & 0.38 & 0.947 \\
\hline \multicolumn{7}{|c|}{ Sc galaxies } \\
\hline$\overline{\text { All }}$ & 1843 & $1.525 \pm 0.032$ & $9.113 \pm 0.022$ & $0.2296 \pm 0.0039$ & 0.31 & 0.871 \\
\hline Sparse & 1098 & $1.472 \pm 0.038$ & $9.122 \pm 0.025$ & $0.2253 \pm 0.0052$ & 0.28 & 0.847 \\
\hline Rich & 243 & $1.565 \pm 0.091$ & $9.146 \pm 0.067$ & $0.2248 \pm 0.01$ & 0.41 & 0.887 \\
\hline Rich $N>10$ & 112 & $1.554 \pm 0.13$ & $9.134 \pm 0.091$ & $0.2139 \pm 0.014$ & 0.38 & 0.887 \\
\hline Rich BCG N $>10$ & 8 & $0.6245 \pm 0.3$ & $10.09 \pm 0.25$ & $0.08414 \pm 0.025$ & 0.55 & 0.893 \\
\hline Rich satellite $N \geq 10$ & 104 & $1.498 \pm 0.15$ & $9.151 \pm 0.1$ & $0.2074 \pm 0.015$ & 0.38 & 0.884 \\
\hline Red sequence & 409 & $1.661 \pm 0.078$ & $8.929 \pm 0.052$ & $0.2133 \pm 0.007$ & 0.36 & 0.893 \\
\hline Blue cloud & 1434 & $1.53 \pm 0.037$ & $9.138 \pm 0.025$ & $0.2261 \pm 0.0051$ & 0.3 & 0.865 \\
\hline
\end{tabular}


TABLE 4

SERSIC LUMINOSITY- EFFECTIVE RADII RELATIONSHIPS

\begin{tabular}{|c|c|c|c|c|c|c|}
\hline Class & $\mathrm{N}$ & $\begin{array}{c}\text { Slope } \\
{\left[\log \left(L_{\odot}\right)\right]}\end{array}$ & $\begin{array}{c}\text { Intercept } \\
{\left[\frac{\log \left(L_{\odot}\right)}{\log (\mathrm{kpc})}\right]}\end{array}$ & $\begin{array}{l}\text { Dispersion } \\
{\left[\log \left(L_{\odot}\right)\right]}\end{array}$ & $\begin{array}{c}\text { - Interquanti } \\
10 \% \text { quantile } \\
{[\log (\mathrm{kpc})]}\end{array}$ & $\begin{array}{c}\text { Size Range - } \\
90 \% \text { quantile } \\
{[\log (\mathrm{kpc})]}\end{array}$ \\
\hline \multicolumn{7}{|c|}{ Elliptical galaxies } \\
\hline $\begin{array}{r}\text { All } \\
\text { Sparse } \\
\text { Rich } \\
\text { Rich } \mathrm{N} \geq 10 \\
\text { Rich BCG N } \geq 10 \\
\text { Rich satellite } \mathrm{N} \geq 10 \\
\text { Red sequence } \\
\text { Blue cloud }\end{array}$ & $\begin{array}{c}3156 \\
800 \\
1509 \\
764 \\
374 \\
390 \\
2904 \\
252\end{array}$ & $\begin{array}{c}1.379 \pm 0.015 \\
1.364 \pm 0.03 \\
1.311 \pm 0.022 \\
1.304 \pm 0.022 \\
1.098 \pm 0.04 \\
1.266 \pm 0.036 \\
1.403 \pm 0.013 \\
1.176 \pm 0.06\end{array}$ & $\begin{array}{c}9.639 \pm 0.011 \\
9.634 \pm 0.018 \\
9.698 \pm 0.017 \\
9.707 \pm 0.017 \\
9.9 \pm 0.034 \\
9.707 \pm 0.023 \\
9.62 \pm 0.0093 \\
9.803 \pm 0.041\end{array}$ & $\begin{array}{c}0.1453 \pm 0.0027 \\
0.1646 \pm 0.0047 \\
0.1281 \pm 0.0031 \\
0.1238 \pm 0.0042 \\
0.1026 \pm 0.0035 \\
0.1347 \pm 0.0065 \\
0.1386 \pm 0.0025 \\
0.1963 \pm 0.013\end{array}$ & $\begin{array}{l}0.24 \\
0.14 \\
0.33 \\
0.28 \\
0.58 \\
0.23 \\
0.25 \\
0.14\end{array}$ & $\begin{array}{c}0.945 \\
0.838 \\
0.985 \\
1.01 \\
1.05 \\
0.875 \\
0.947 \\
0.893\end{array}$ \\
\hline \multicolumn{7}{|c|}{ So galaxies } \\
\hline $\begin{array}{r}\text { All } \\
\text { Sparse } \\
\text { Rich } \\
\text { Rich } \mathrm{N} \geq 10 \\
\text { Rich BCG } \mathrm{N} \geq 10 \\
\text { Rich satellite } \mathrm{N} \geq 10 \\
\text { Red sequence } \\
\text { Blue cloud }\end{array}$ & $\begin{array}{c}1887 \\
749 \\
561 \\
306 \\
32 \\
274 \\
1518 \\
369\end{array}$ & $\begin{array}{c}1.24 \pm 0.019 \\
1.195 \pm 0.037 \\
1.278 \pm 0.033 \\
1.224 \pm 0.046 \\
0.7051 \pm 0.12 \\
1.172 \pm 0.052 \\
1.217 \pm 0.026 \\
1.231 \pm 0.05\end{array}$ & $\begin{array}{c}9.662 \pm 0.01 \\
9.69 \pm 0.018 \\
9.629 \pm 0.019 \\
9.631 \pm 0.027 \\
10.11 \pm 0.07 \\
9.638 \pm 0.025 \\
9.656 \pm 0.013 \\
9.738 \pm 0.03\end{array}$ & $\begin{array}{c}0.1791 \pm 0.0037 \\
0.172 \pm 0.0057 \\
0.1819 \pm 0.0054 \\
0.1759 \pm 0.007 \\
0.1036 \pm 0.011 \\
0.173 \pm 0.0068 \\
0.1711 \pm 0.0042 \\
0.1931 \pm 0.0079\end{array}$ & $\begin{array}{l}0.15 \\
0.13 \\
0.19 \\
0.17 \\
0.36 \\
0.16 \\
0.16 \\
0.14\end{array}$ & $\begin{array}{c}0.807 \\
0.777 \\
0.826 \\
0.77 \\
0.855 \\
0.764 \\
0.78 \\
0.844\end{array}$ \\
\hline \multicolumn{7}{|c|}{ Sa galaxies } \\
\hline $\begin{array}{r}\text { All } \\
\text { Sparse } \\
\text { Rich } \\
\text { Rich } \mathrm{N} \geq 10 \\
\text { Rich BCG } \mathrm{N} \geq 10 \\
\text { Rich satellite } \mathrm{N} \geq 10 \\
\text { Red sequence } \\
\text { Blue cloud }\end{array}$ & $\begin{array}{c}1952 \\
882 \\
483 \\
237 \\
22 \\
215 \\
999 \\
953\end{array}$ & $\begin{array}{c}1.189 \pm 0.019 \\
1.194 \pm 0.032 \\
1.144 \pm 0.041 \\
1.054 \pm 0.064 \\
0.857 \pm 0.21 \\
1.002 \pm 0.07 \\
1.067 \pm 0.032 \\
1.296 \pm 0.033\end{array}$ & $\begin{array}{c}9.627 \pm 0.013 \\
9.618 \pm 0.02 \\
9.669 \pm 0.028 \\
9.682 \pm 0.039 \\
9.948 \pm 0.17 \\
9.7 \pm 0.044 \\
9.675 \pm 0.02 \\
9.578 \pm 0.023\end{array}$ & $\begin{array}{c}0.1776 \pm 0.0038 \\
0.1687 \pm 0.0048 \\
0.1765 \pm 0.0063 \\
0.1884 \pm 0.0085 \\
0.1316 \pm 0.015 \\
0.1893 \pm 0.0094 \\
0.1834 \pm 0.0048 \\
0.1661 \pm 0.0048\end{array}$ & $\begin{array}{c}0.22 \\
0.22 \\
0.22 \\
0.22 \\
0.51 \\
0.22 \\
0.2 \\
0.3\end{array}$ & $\begin{array}{c}0.889 \\
0.871 \\
0.911 \\
0.901 \\
0.959 \\
0.862 \\
0.87 \\
0.903\end{array}$ \\
\hline \multicolumn{7}{|c|}{ Sb galaxies } \\
\hline $\begin{array}{r}\text { All } \\
\text { Sparse } \\
\text { Rich } \\
\text { Rich } \mathrm{N} \geq 10 \\
\text { Rich BCG } \mathrm{N} \geq 10 \\
\text { Rich satellite } \mathrm{N} \geq 10 \\
\text { Red sequence } \\
\text { Blue cloud }\end{array}$ & $\begin{array}{c}2733 \\
1279 \\
614 \\
263 \\
36 \\
227 \\
788 \\
1945\end{array}$ & $\begin{array}{c}1.314 \pm 0.02 \\
1.303 \pm 0.031 \\
1.355 \pm 0.041 \\
1.311 \pm 0.066 \\
0.6799 \pm 0.16 \\
1.245 \pm 0.077 \\
1.185 \pm 0.034 \\
1.374 \pm 0.023\end{array}$ & $\begin{array}{c}9.452 \pm 0.015 \\
9.453 \pm 0.023 \\
9.444 \pm 0.032 \\
9.437 \pm 0.053 \\
10.11 \pm 0.14 \\
9.458 \pm 0.054 \\
9.501 \pm 0.025 \\
9.426 \pm 0.018\end{array}$ & $\begin{array}{c}0.1795 \pm 0.0028 \\
0.1763 \pm 0.0039 \\
0.1796 \pm 0.0068 \\
0.1893 \pm 0.01 \\
0.1251 \pm 0.015 \\
0.1838 \pm 0.01 \\
0.1941 \pm 0.0053 \\
0.1699 \pm 0.0031\end{array}$ & $\begin{array}{c}0.37 \\
0.35 \\
0.4 \\
0.39 \\
0.59 \\
0.38 \\
0.28 \\
0.4\end{array}$ & $\begin{array}{c}0.958 \\
0.938 \\
0.99 \\
0.952 \\
1.04 \\
0.926 \\
0.974 \\
0.95\end{array}$ \\
\hline $\begin{array}{r}\text { Sc galaxies All } \\
\text { Sparse } \\
\text { Rich } \\
\text { Rich } N \geq 10 \\
\text { Rich BCG } N \geq 10 \\
\text { Rich satellite } \mathrm{N} \geq 10 \\
\text { Red sequence } \\
\text { Blue cloud }\end{array}$ & $\begin{array}{c}1843 \\
1098 \\
243 \\
112 \\
8 \\
104 \\
409 \\
1434\end{array}$ & $\begin{array}{c}1.561 \pm 0.03 \\
1.509 \pm 0.037 \\
1.606 \pm 0.095 \\
1.593 \pm 0.14 \\
0.6577 \pm 0.28 \\
1.538 \pm 0.14 \\
1.704 \pm 0.079 \\
1.567 \pm 0.035\end{array}$ & $\begin{array}{c}9.093 \pm 0.02 \\
9.102 \pm 0.025 \\
9.12 \pm 0.067 \\
9.11 \pm 0.1 \\
10.07 \pm 0.23 \\
9.127 \pm 0.099 \\
8.902 \pm 0.054 \\
9.118 \pm 0.023\end{array}$ & $\begin{array}{c}0.2285 \pm 0.0042 \\
0.2242 \pm 0.0051 \\
0.2238 \pm 0.0096 \\
0.2125 \pm 0.015 \\
0.08345 \pm 0.023 \\
0.206 \pm 0.014 \\
0.2111 \pm 0.0081 \\
0.2249 \pm 0.0046\end{array}$ & $\begin{array}{c}0.32 \\
0.28 \\
0.43 \\
0.39 \\
0.56 \\
0.39 \\
0.36 \\
0.3\end{array}$ & $\begin{array}{l}0.874 \\
0.848 \\
0.889 \\
0.888 \\
0.904 \\
0.886 \\
0.893 \\
0.867\end{array}$ \\
\hline
\end{tabular}


TABLE 5

DE VAUCOULEURS LUMINOSITY-SIZE RELATIONSHIPS

\begin{tabular}{|c|c|c|c|c|c|c|}
\hline Class & $\mathrm{N}$ & $\begin{array}{c}\text { Slope } \\
{\left[\log \left(L_{\odot}\right)\right]}\end{array}$ & $\begin{array}{c}\text { Intercept } \\
{\left[\frac{\log \left(L_{\odot}\right)}{\log (\mathrm{kpc})}\right]}\end{array}$ & $\begin{array}{l}\text { Dispersion } \\
{\left[\log \left(L_{\odot}\right)\right]}\end{array}$ & $\begin{array}{c}\text { - Interquantile } \\
10 \% \text { quantile } \\
{[\log (\mathrm{kpc})]}\end{array}$ & $\begin{array}{l}\text { Size Range - } \\
90 \% \text { quantile } \\
{[\log (\mathrm{kpc})]}\end{array}$ \\
\hline \multicolumn{7}{|c|}{ E galaxies } \\
\hline All & 3162 & $1.1 \pm 0.014$ & $9.734 \pm 0.011$ & $0.1821 \pm 0.0036$ & 0.3 & 1.15 \\
\hline Sparse & 801 & $1.058 \pm 0.033$ & $9.727 \pm 0.022$ & $0.2004 \pm 0.0061$ & 0.21 & 1.02 \\
\hline Rich & 1514 & $1.054 \pm 0.015$ & $9.8 \pm 0.014$ & $0.158 \pm 0.0049$ & 0.41 & 1.24 \\
\hline Rich $N \geq 10$ & 766 & $1.064 \pm 0.022$ & $9.789 \pm 0.02$ & $0.1553 \pm 0.0062$ & 0.39 & 1.31 \\
\hline Rich BCG $N \geq 10$ & 374 & $0.8154 \pm 0.03$ & $10.08 \pm 0.031$ & $0.1186 \pm 0.0046$ & 0.67 & 1.41 \\
\hline Rich satellite $\mathrm{N} \geq 10$ & 392 & $1.042 \pm 0.037$ & $9.765 \pm 0.027$ & $0.1659 \pm 0.0098$ & 0.27 & 1.03 \\
\hline Red sequence & 2907 & $1.137 \pm 0.013$ & $9.704 \pm 0.011$ & $0.1747 \pm 0.0037$ & 0.32 & 1.15 \\
\hline Blue cloud & 255 & $0.8198 \pm 0.06$ & $9.97 \pm 0.047$ & $0.2315 \pm 0.013$ & 0.19 & 1.13 \\
\hline
\end{tabular}

